\title{
Sliver Doped Sodium Antimonate with Greatly Reduced the Band Gap for Efficiently Enhanced Photocatalytic Activities Under Visible Light (Experiment and DFT Calculation)
}

\author{
Chen Chen ${ }^{a . b *}\left(\mathbb{D}\right.$, Lei Wang ${ }^{a}$, Ting Cheng ${ }^{c}$, Xiao Zhang ${ }^{b}$, Yuan Tian ${ }^{a}$, YiSu Shi ${ }^{a}$ \\ ${ }^{a}$ Jiangsu University of Science and Technology, School of Environmental and Chemical Engineering, \\ Zhenjiang, P. R. China. \\ ${ }^{b}$ Nanjing University and Yancheng Academy of Environmental Technology and Engineering, Yancheng, \\ P. R. China. \\ 'School of Environmental Ecology, Jiangsu City Vocational College, P. R. China.
}

Received: February 24, 2021; Revised: July 18, 2021; Accepted: August 1, 2021

In this work, $\mathrm{Ag}$ doped $\mathrm{NaSbO}_{3}$ (ANS) was successfully synthesized and applied an efficient visible light photocatalyst. The properties of catalyst were fully characterized, and DFT (the density functional theory) computation was utilized to investigate the photocatalytic mechanism. The various characterization findings proved that $\mathrm{Ag}$ atoms were perfectly doped into $\mathrm{NaSbO}_{3}$ crystals, which greatly reduced the band gap, and made ANS catalyst exhibit excellent visible light absorption performance. In addition, ANS appeared great photocatalytic activities and remarkable stability. Also, electrochemical characterizations illustrated that ANS possessed stronger ability of generating and transferring electrons and holes under visible light, and the doping of $\mathrm{Ag}$ atoms reduced the resistance of charge transfer, thus effectively accelerated the photocatalysis reaction. Moreover, through ESR characterization and scavengers coexistent experiments, $\bullet \mathrm{OH}$ and $\bullet \mathrm{O}_{2}^{-}$were considered to be active radicals that played a crucial role in the photodegradation process. Furthermore, DFT calculation demonstrated that ANS displayed obvious visible light excitation performance via reducing the band gap. The improvement of work function enhanced the oxidation ability of photogenerated holes, forming more hydroxyl radicals, thus boosted the photodegradation of pollutants.

Keywords: Sliver Doping, Sodium Antimonate, Photocatalyst, Band gap, DFT computation.

\section{Introduction}

With the continuous development of the chemical industry, the amount of organic wastewater discharged by enterprises is increasing year by year ${ }^{1-3}$. If this kind of wastewater is discharged directly or treated improperly, it would cause serious harm to environmental safety and human health. At present, many techniques, including biological process ${ }^{4,5}$, physical chemical combination method $^{3,6}$, adsorption ${ }^{7-9}$, membrane separation ${ }^{10,11}$, advanced oxidation $^{12-14}$, photocatalytic degradation ${ }^{15,16}$, could be used to treat organic wastewater. Each of these approaches has its own advantages and disadvantages. For example, biotechnology is relatively mature for wastewater treatment, but it has strict requirements on the influent concentration and treatment conditions. The physicochemical method is effective to degrade pollutants. However, it requires a large amount of chemical agents, and is prone to produce by-products during the process of treatment. The treatment device of adsorption is easy to operate, but there are difficulties in the reuse of adsorbent and treatment of waste adsorbent. The membrane separation technique exhibits a favorable initial

*e-mail: chenc@just.edu.cn treatment effect, while the post-treatment efficiency is prone to decrease after membrane fouling. The advanced oxidation technique can remove a wide range of pollutants, whereas the treatment cost is extremely high. The photocatalytic degradation is a new type of water treatment technology developed in the last century ${ }^{14,17}$. It has many advantages, such as simple equipment, high treatment efficiency, and long service life of catalyst.

According to the classical theory of energy bands in solid, there are valence bands and conduction bands in electron energy bands ${ }^{18,19}$. The energy difference between the valence band top and the conduction band bottom is called band gap. When the band gap is ranged from $1.5 \mathrm{eV}$ to $3.0 \mathrm{eV}$, the material will have obvious visible light absorption properties ${ }^{20,21}$. When a solid with suitable band gap absorbs visible light, electrons in the valence band can be excited to the conduction band to form photogenic electrons and photonic holes in the valence band. After these photogenerated electrons and photogenerated holes diffuse to the solid surface, they can not only directly oxidize and reduce efficiently with pollutants, but also react with oxygen and hydroxyl radicals in system to form highly active superoxide radicals and hydroxyl radicals, and further decompose pollutants ${ }^{22,23}$. 
At present, the main catalysts for photocatalytic reactions under visible light include $\mathrm{ZnO}^{24,}{ }^{25}, \mathrm{BiVO}_{4}{ }^{26,27}, \mathrm{KNbO}_{3}{ }^{28}$, $\mathrm{CdS}^{29,30}, \mathrm{C}_{3} \mathrm{~N}_{4}{ }^{31}, \mathrm{Ag}_{3} \mathrm{PO}_{4}{ }^{32,33}, \mathrm{AgBr}^{34}$ and so forth. Sb element related materials including $\mathrm{BiSbO}_{4} / \mathrm{BiOBr}$ nanoarchitecture ${ }^{35}$, $\mathrm{AgI} / \mathrm{BiSbO}_{4}$ heterojunction ${ }^{36}$ and carbon dots modified $\mathrm{BiSbO}_{4}$ composites $^{37}$ have attracted much attention in the field of photocatalysis. During $\mathrm{Sb}$ related materials, $\mathrm{NaSbO}_{3}$ is a common industrial raw material, which can be used as opaque filler, enamel whitening agent, iron sheet, and acid resistant paint of steel plate, etc. It can be also used as a raw material for the synthesis of $\mathrm{AgSbO}_{3}{ }^{38,39}$. In addition, $\mathrm{NaSbO}_{3}$ itself displays certain photosensitivity. However, because its band gap is as high as about $4.5 \mathrm{eV}$, so only ultraviolet light can excite electrons in its valence band to the conduction band. This greatly limits the application of $\mathrm{NaSbO}_{3}$ in the field of photocatalysis ${ }^{40,41}$. Theoretically speaking, each photocatalyst has its own specific electron energy band. However, due to its limited light absorption and critical problems of photogenerated holes recombination caused by poor charge separation, the reducing capability of a single semiconductor photocatalytic material are relatively limited. In this situation, many researchers have focused their efforts on various composite photocatalytic materials, to address these issues and thus accelerate the photocatalytic activities, and then enhance the photocatalytic degradation efficiencies ${ }^{42,43}$. According to the theory of semiconductor physics, impurity doping is an effective method to decrease the band gap of semiconductor materials ${ }^{44,45}$. Appropriate impurity doping could add additional energy levels between the valence band and conduction band of raw material, effectively reduce the band gap of semiconductor, and make it excited under visible light, thus improve the photocatalytic degradation performance ${ }^{25,46}$. If composite photocatalysts can decrease the band gap through the doping, then enhancement of photocatalytic performance is expected.

In this work, the Ag atom was successfully doped into $\mathrm{NaSbO}_{3}$ crystals by hydrothermal ion exchange reaction, and prepared $\mathrm{Ag}$ doped $\mathrm{NaSbO}_{3}$ visible light photocatalytic material (ANS). Our research firstly demonstrated that simple doping of photocatalyst could highly reduce the band gap of material, making photocatalyst exhibit excellent absorption performance under visible light, and enhancing the oxidation ability of photogenerated holes. The various properties of catalyst were fully characterized by modern analytical instruments, including its chemical composition, morphology, doping structure, optical absorption, and so forth. Also, the photodegradation mechanism of ANS catalyst was illustrated with the aid of a series of electrochemical tests. Moreover, the VASP software and DFT calculation was conducted to further investigate the action of photogenerated holes, and deeply clarify the photocatalytic mechanism. Besides, the photocatalytic efficiency of ANS was evaluated by photodegradation experiments of Methylene Blue (MB) in solution. Additionally, to clarify the reaction processes, the impact factors, including catalyst dosage, reaction time, reaction kinetics, trapping agents, and recycle performance were studied. Furthermore, this technique is highly useful in designing more capable photocatalysts for various applications in the photodegradation of organic pollutants.

\section{Experiments and Methods}

\subsection{Synthesis of photocatalyst}

The synthesis steps of photocatalyst were as follows: Firstly, $0.0035 \mathrm{~mol}$ of $\mathrm{NaSbO}_{3} \cdot 3 \mathrm{H}_{2} \mathrm{O}$ and $0.0010 \mathrm{~mol}$ to $0.0035 \mathrm{~mol}$ of $\mathrm{AgNO}_{3}$ were weighed, respectively. $\mathrm{NaSbO}_{3} \cdot 3 \mathrm{H}_{2} \mathrm{O}$ was added into $60 \mathrm{ml}$ deionized water to form a milky white hydrosol system. Secondly, $\mathrm{AgNO}_{3}$ was gradually added into the mixture while stirred with magnetic force. Thirdly, the mixture was stirred at the temperature of $55^{\circ} \mathrm{C}$ for 90 minutes to form a coffee color hydrosol system. Fourthly, this mixture was transferred to a stainless steel reactor on a teflon substrate for hydrothermal reaction for 9 hours at $90^{\circ} \mathrm{C}$. After the reaction was completed, the reaction product was then cooled to room temperature (the air conditioning in laboratory was set at $25^{\circ} \mathrm{C}$ ), filtered, washed with deionized water for several times until its neutral. After that, the reaction product was dried at $75^{\circ} \mathrm{C}$ in the blast drying oven. Finally, the synthetic product was grounded into finer particles with an agate mortar, and the photocatalyst was obtained. The schematic illustration of the synthesis process was depicted in Figure 1.
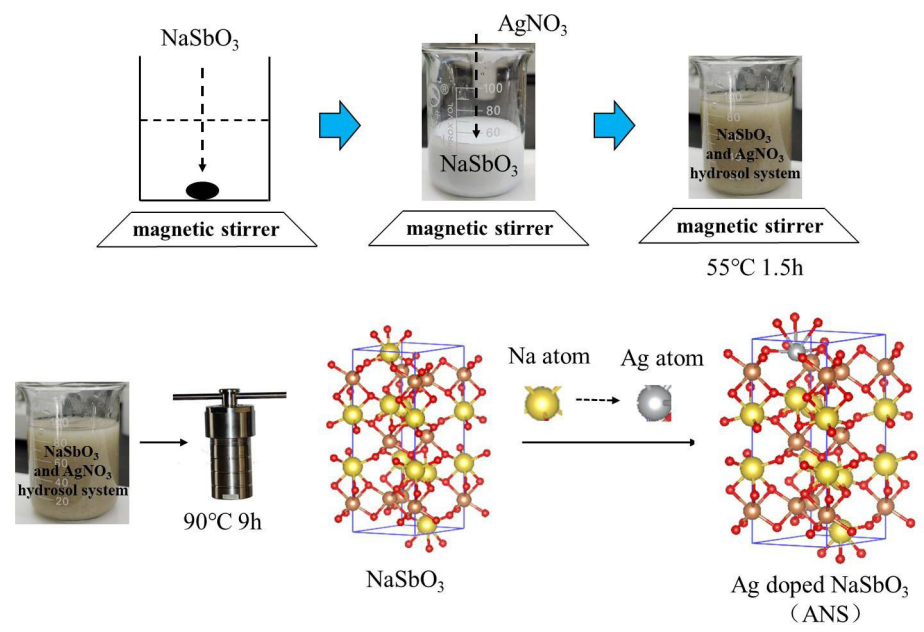

Figure 1. The schematic illustration of the synthesis process. 
Figure 2 depicted the effect of the molar ratio of $\mathrm{AgNO}_{3}$ and $\mathrm{NaSbO}_{3}$ on the removal rate of $\mathrm{MB}$ (30 minutes) during the synthesis process. As the molar ratio increased, the degradation effect of MB was gradually improved. However, when the molar ratio increased to 0.57 , the degradation effect of MB was no longer obvious. Therefore, considering the cost of the ANS catalyst, it was believed that the molar ratio of 0.57 was more economical.

\subsection{Materials characterization}

To illustrate the various properties of material synthesized, samples were fully characterized by Scanning electron microscopy-energy dispersive spectroscopy (SEM-EDX), X-ray diffraction (XRD), X-ray photoelectron spectroscopy (XPS), UV-vis diffuse reflectance spectra, photoluminescence (PL), Electron spin resonance (ESR), electrochemical impedance spectroscopy (EIS), electrochemical photocurrent (interval 20 s), and other advanced instruments. The Liquid specific surface area of ANS Catalyst was analyzed through Xigo Liquid specific surface area Determinator. The particle size distribution was analyzed by Malvern Mastersizer 2000 Laser particle size analyzer. The morphologies of material were examined with a HITACHI(S-3400N) scanning electron microscope. XRD patterns of powder samples were recorded by a Shimadzu XD-3A diffractometer, employing $\mathrm{Cu}-\mathrm{K} \alpha$ radiation $(\lambda=1.54056 \AA)$. The $\mathrm{X}$-ray photoelectron spectroscopy spectra were examined by a PHI 5000 VersaProbe XPS equipment. The UV-vis spectra were conducted by PerkinElmer Ultraviolet spectrophotometer. The solid photoluminescence was conducted by HITACHI Fluorescence spectrometer (F7000). The Electron spin resonance was examined by Electron paramagnetic resonance spectrometer (EMXmicro-6/1/P/L, Karlsruhe, Germany utilizing). The DMPO (5, 5-dimethyl-1pyrroline $\mathrm{N}$-oxide) was used as free radical $\left(\cdot \mathrm{O}_{2}^{-}\right.$and $\left.\bullet \mathrm{OH}\right)$ trapping agent. The electrochemical impedance spectroscopy and photocurrent (interval 20s) was measured on a CHI 660E electrochemical workstation.

\subsection{Photocatalytic experiments of ANS}

Photocatalytic experiments were conducted through degradation of methylene blue under visible light irradiation

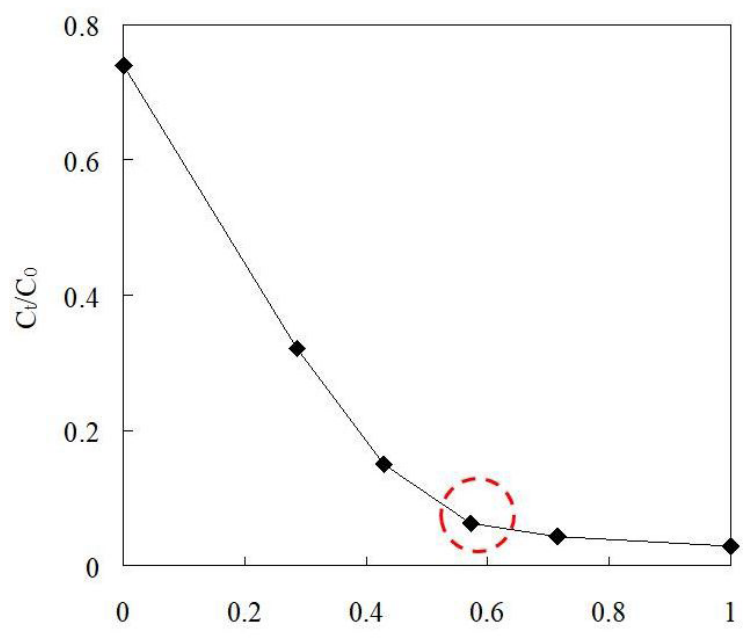

The mole ratio $\left(\mathrm{AgNO}_{3} / \mathrm{NaSbO}_{3}\right)$

Figure 2. The effect of mole ratio $\left(\mathrm{AgNO}_{3} / \mathrm{NaSbO}_{3}\right)$ in synthesis process on the removal rate of MB.
(300W Xe-lamp). During each experiment, the photocatalyst (from $0.005 \mathrm{~g}$ to $0.030 \mathrm{~g}$ ) was added into $30 \mathrm{ml} \mathrm{MB}$ solution (the initial concentration was $10 \mathrm{mg} / \mathrm{L}$ ). All photocatalytic experiments were conducted at room temperature (the air conditioning in laboratory was set at $25^{\circ} \mathrm{C}$ ), and the mixtures were put into a transparent photoreaction vessel. In addition, the photocatalytic reaction time ranged from 0 to 30 minutes. After the reaction, the concentration of $\mathrm{MB}$ was determined by Ultraviolet visible spectrophotometer at $662 \mathrm{~nm}$.

To estimate the photodegradation performance of recycled sample, the consecutive cycles were done to measure the photocatalytic efficiencies of MB. At the same time, the morphology and XRD patterns of recycled ANS were also investigated. After each recycled cycle, the catalyst was filtered out from the solution by $0.45 \mu \mathrm{m}$ filter membrane, and the filer solids were washed by deionized water for several times. Then the sample was dried to constant weight at a temperature of $75^{\circ} \mathrm{C}$ in the oven for the next cycle. To analyze the active species generated in the process of photocatalytic degradation reaction and illustrate the possible mechanism, different trapping agents were put into the reaction mixtures. These scavenger making use of quenching reactive species include EDTA-2Na, TBA (tert-butyl alcohol), BQ (1,4-benzoquinone), and $\mathrm{CCl}_{4}$.

\subsection{DFT calculation}

The DFT calculation process was conducted through Vienna Ab initio Simulation Package (VASP) software ${ }^{47}$ and spin-polarized Density Functional Theory (DFT). The exchange-correlation potential was calculated by PerdewBurke-Ernzerhof functional $(\mathrm{PBE})^{48,49}$ within the generalized gradient approximation (GGA) method $\mathrm{d}^{50,51}$. In the process of calculation, the cut-off energy was $450 \mathrm{eV}$, and the K-points grids of dimensions were $5 \times 5 \times 5$. Figure 3 displayed the
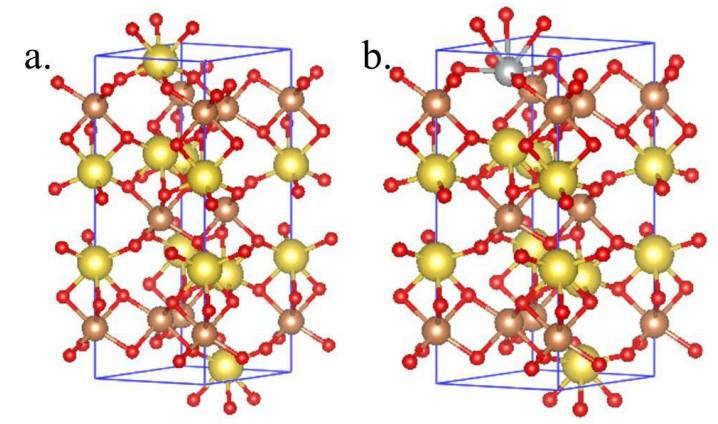

c.
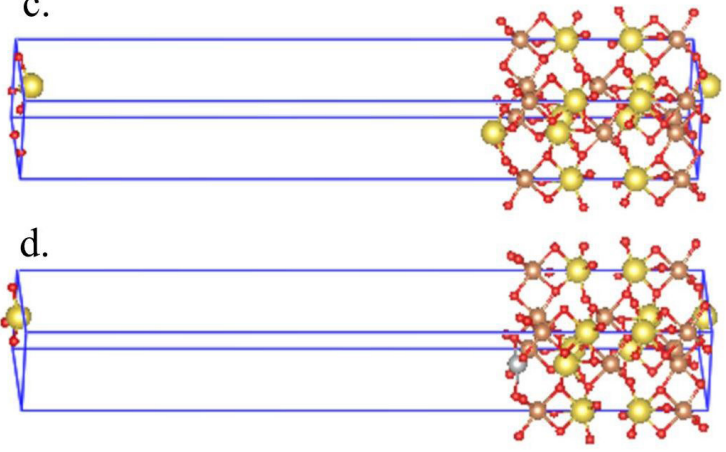

Figure 3. The theory structure of crystal model $\mathrm{NaSbO}_{3}$ (a) and ANS Catalyst (b), and the theory structure of surface model (for work function calculation) $\mathrm{NaSbO}_{3}$ (c) and ANS catalyst (d) (yellow ball is $\mathrm{Na}$ atom, coffee ball is $\mathrm{Sb}$ atom, red ball is $\mathrm{O}$ atom, grey ball is $\mathrm{Ag}$ atom). 
theory structure of crystal $\mathrm{NaSbO}_{3}$ and ANS catalyst (Ag doped $\mathrm{NaSbO}_{3}$ ), the surface model of $\mathrm{NaSbO}_{3}$ and ANS catalyst. In addition, we calculated the band structure and DOS using crystal model of $\mathrm{NaSbO}_{3}$ (Figure 3a) and ANS catalyst (Figure 3b). We also computed the work function through the surface model of $\mathrm{NaSbO}_{3}$ (Figure $3 \mathrm{c}$ ) and ANS catalyst (Figure $3 \mathrm{~d}$ ). The vacuum slab of surface model was designed as $30 \AA$ for both $\mathrm{NaSbO}_{3}$ and ANS catalyst. First of all, both the atomic coordinates and cell parameters of $\mathrm{NaSbO}_{3}$ and ANS catalyst were optimized in the process of calculation, respectively. The theoretical crystal structure of $\mathrm{NaSbO}_{3}$ is a cell unit, including $6 \mathrm{Na}, 6 \mathrm{Sb}$ and $18 \mathrm{O}$ atoms. The theoretical crystal structure of ANS catalyst is a cell unit, including $1 \mathrm{Ag}, 5 \mathrm{Na}, 6 \mathrm{Sb}$, and $18 \mathrm{O}$ atoms.

\section{Results and Discussions}

\subsection{Particle size and liquid specific surface area}

Figure $4 \mathrm{a}$ described the particle size of ANS Catalyst. It was observed that the particle size of ANS Catalyst ranged from $240 \mathrm{~nm}$ to $6.607 \mathrm{um}\left(\mathrm{d}_{0.1}=705 \mathrm{~nm}, \mathrm{~d}_{0.5}=2.01 \mathrm{um}\right.$, $\left.\mathrm{d}_{0.9}=4.37 \mathrm{um}\right)$. Figure $4 \mathrm{~b}$ depicted the analytical results of the liquid-phase surface area for ANS Catalyst. According to the fitting calculation of instrument software, the liquid specific surface area of ANS Catalyst was about $543.7 \mathrm{~m}^{2} / \mathrm{g}$.

\section{2. $X R D$}

Figure 5 described XRD patterns of original $\mathrm{NaSbO}_{3}$ and ANS catalyst, respectively. For original $\mathrm{NaSbO}_{3}$, the main peaks could be found at $2 \theta, 14.9^{\circ}, 16.6^{\circ}, 20.1^{\circ}, 20.3^{\circ}$, $28.8^{\circ}, 29.4^{\circ}, 29.6^{\circ}, 30.2^{\circ}, 33.8^{\circ}, 37.8^{\circ}, 48.4^{\circ}$, and $50.4^{\circ}$. In contrast, there were no obvious variations in the position of the peaks for the ANS Catalyst. This phenomenon indicated that $\mathrm{NaSbO}_{3}$ and $\mathrm{AgSbO}_{3}$ have similar crystal structure and atomic coordinates as theory structure in Figure. $3 \mathrm{a}$ and $3 \mathrm{~b}$. In addition, some peaks of ANS Catalyst including $2 \theta$ at about $14.9^{\circ}, 16.6^{\circ}, 20.1^{\circ}, 20.3^{\circ}, 37.8^{\circ}$ and $48.4^{\circ}$ was obviously lower than that of $\mathrm{NaSbO}_{3}$. These results implied that the doping of Ag made the crystals of ANS Catalyst materials appear deformities and defects, resulting in the decrease of crystal integrity ${ }^{52-54}$.
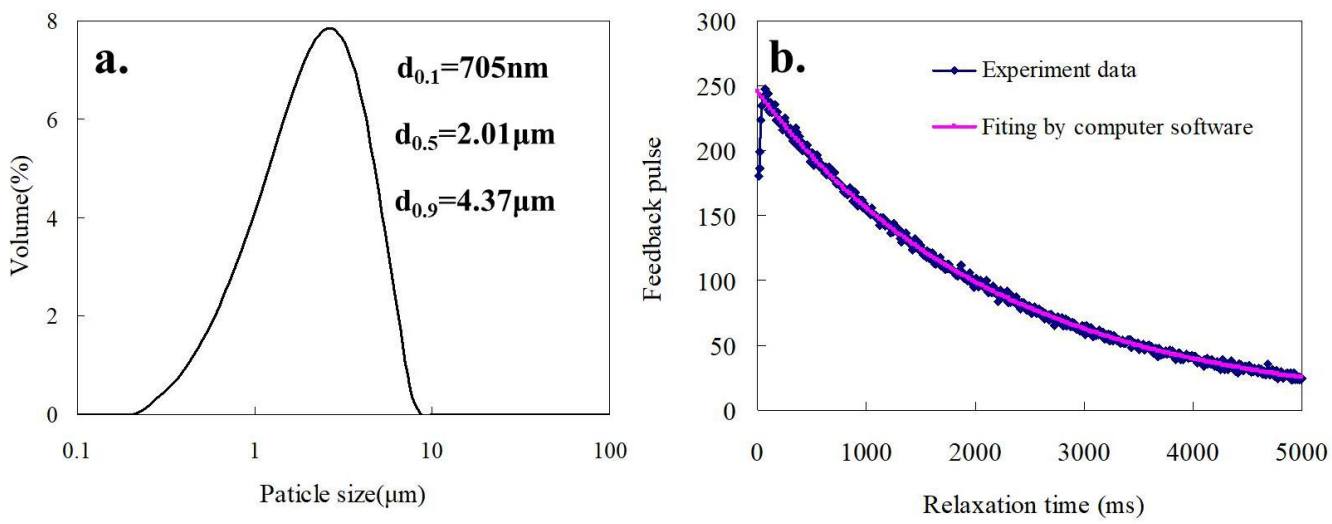

Figure 4. Particle size distribution and liquid specific surface area results of ANS Catalyst.

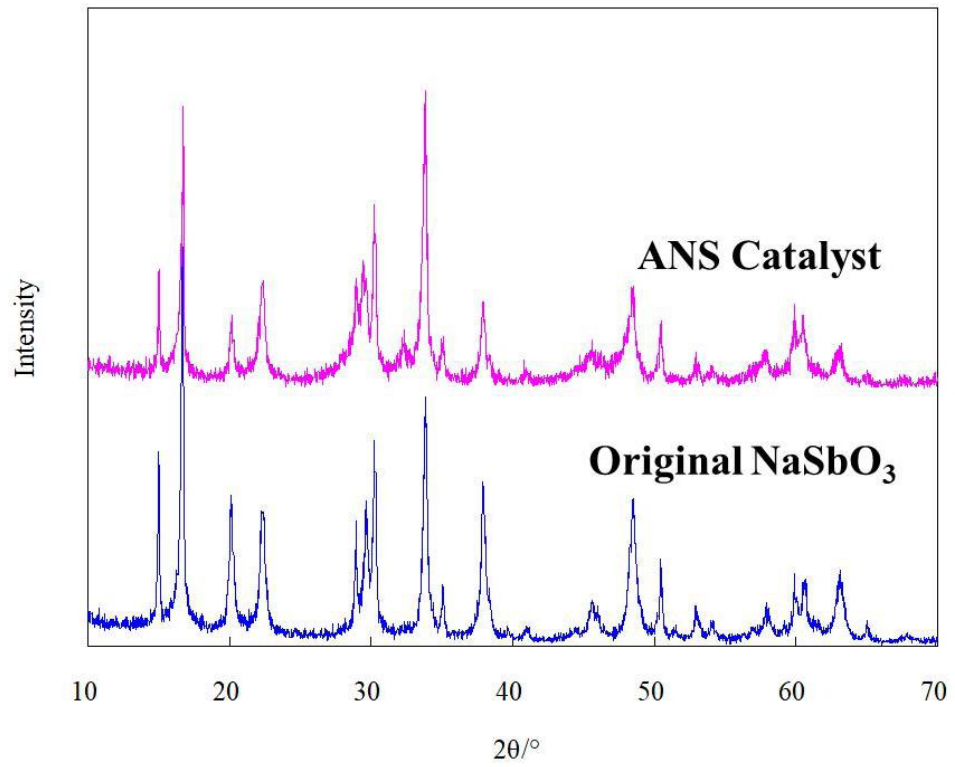

Figure 5. XRD patterns of original $\mathrm{NaSbO}_{3}$ and $\mathrm{ANS}$ Catalyst. 


\subsection{UV-vis diffuse reflectance spectra}

The results of UV-vis diffuse reflectance spectra were depicted in Figure 6. It could be observed that the broad absorption bands of $\mathrm{NaSbO}_{3}$ were approximately $300 \mathrm{~nm}$, while that of the ANS catalyst were 520nm. These findings indicated that ANS catalyst exhibited better visible light absorption than $\mathrm{NaSbO}_{3}$ after doping with $\mathrm{Ag}$ atom. For the calculation of band gap energy, Tauc equation is often used as follows:

$\alpha(v) h v=A(h v-E g)^{n / 2}$

where, $\alpha, \mathrm{h}, \mathrm{v}, \mathrm{A}$ and $\mathrm{Eg}$ represents absorption coefficient at light frequency v, Planck constant, light frequency, a constant and band gap energy, respectively. For direct transition semiconductor as $\mathrm{NaSbO}_{3}$, the value of $\mathrm{n}$ is 1 .

According to Tauc equation, the value of Eg could be obtained through plotting the curve of $[a(v) h v]^{2}$ versus $h v$, and extra-polating the linear part of the curve to zero absorption coefficient. This analysis process for $\mathrm{NaSbO}_{3}$ and ANS catalyst was conducted, and the related findings were described in Figure $6 \mathrm{~b}$. We observed that the band gaps energy of $\mathrm{NaSbO}_{3}$ was $4.2 \mathrm{eV}$, which was very similar to that of former reports ${ }^{40}$. Moreover, the band $\mathrm{NaSbO}_{3}$ gap energy of ANS catalyst was $2.3 \mathrm{eV}$, which was much smaller than $\mathrm{NaSbO}_{3}$. These findings indicated that the doping of Ag atom could greatly reduce the band gap, and make ANS catalyst exhibit excellent absorption performance under visible light.

\subsection{SEM-EDX}

To further observe the microscopic morphology and composition of ANS catalyst, the SEM and EDX of sample was analyzed, and the results were depicted in Figure $7 \mathrm{a}$ to Figure $7 \mathrm{c}$. It could be seen that ANS catalyst presented amorphous morphology. Besides, the EDX results (area 1) indicated that the main chemical composition (atomic percent $/ \%$ ) of ANS catalyst was about $2.47 \%$ Ag, $15.8 \%$ $\mathrm{Na}, 12.9 \% \mathrm{Sb}$, and $68.8 \% \mathrm{O}$. The coexistence of Ag with other elements confirmed that $\mathrm{Ag}$ atom had been doped into $\mathrm{NaSbO}_{3}$ molecules successfully.

Figure $7 \mathrm{~d}$ to Figure $7 \mathrm{~g}$ displayed the EDX element mapping of $\mathrm{Sb}, \mathrm{Ag}, \mathrm{Na}$ and $\mathrm{O}$. It could be observed that the elements ( $\mathrm{Sb}, \mathrm{Ag}, \mathrm{Na}$ and $\mathrm{O}$ ) were mainly distributed in the position where the particles appeared in Figure $7 \mathrm{~b}$.
In addition, there were significant similarities between the distribution of $\mathrm{Ag}$ and other elements through comparing Figure 7d-g. However, the distribution of $\mathrm{Na}$ was looser than that of $\mathrm{O}$ and $\mathrm{Sb}$. This phenomenon could be explained as the replacement of $\mathrm{Na}$ atom by $\mathrm{Ag}$ atom in the crystal structure. In summary, the SEM and EDX analytical results were corresponded to the $\mathrm{Ag}$ doped $\mathrm{NaSbO}_{3}$ catalyst.

\subsection{XPS}

The surface chemical compositions of ANS catalyst were further confirmed by XPS, as shown as Figure 8 . The mainly peaks of ANS catalyst were C1s $(283 \mathrm{eV}), \mathrm{O} 1 \mathrm{~s}$ (530eV), Ag3d (366eV and 372eV), Ag3p (570eV), Sb3d (538eV), Sb3p (768eV and 812eV), Na2s (54eV), and Na2p $(33 \mathrm{eV})$. Except for carbon (testing needs), all the other constituent elements could be found in ANS catalyst, which was corresponded to the results of other characterization techniques. In addition, the more details could be obtained from the high-resolution XPS spectra of O1s and Sb3d (Figure $8 b$ ). After a peak-fitting deconvolution, the strong peak ranging from $525 \mathrm{eV}$ to $534 \mathrm{eV}$ could be decomposed into two individual peaks ( $529 \mathrm{eV}$ and $530 \mathrm{eV})$. The binding energies of $\mathrm{O} 1 \mathrm{~s}$ and $\mathrm{Sb} 3 \mathrm{~d}_{5 / 2}$ were very close. According to the theory area ration between $\mathrm{Sb} 3 \mathrm{~d}_{3 / 2}$ and $\mathrm{Sb} 3 \mathrm{~d}_{5 / 2}$, we speculated that peak located at $530.1 \mathrm{eV}$ was assigned to $\mathrm{O} 1 \mathrm{~s}$, and peak located at $529.1 \mathrm{eV}$ was corresponded to $\mathrm{Sb} 3 \mathrm{~d}_{5 / 2}$. Besides, there also existed the binding energy shift $\left(\mathrm{O} 1 \mathrm{~s}, \mathrm{Sb} 3 \mathrm{~d}_{3 / 2}\right.$ and $\left.\mathrm{Sb} 3 \mathrm{~d}_{5 / 2}\right)$ in the survey spectrum, which might be explained as the bonds between $\mathrm{O}$ and other elements. According to the molecular structure of ANS catalyst, there are two kinds of Sb-O bonds with different bond lengths (1.97 $\AA$ and $1.90 \AA)$, which may cause the binding energy shifts. Additionally, according to the theory peak position and area ration, the peaks of $\mathrm{Ag} 3 \mathrm{~d}_{5 / 2}$ and $\mathrm{Ag} 3 \mathrm{~d}_{3 / 2}$ in Figure $8 \mathrm{c}$ could be obtained at $366 \mathrm{eV}$ and $737 \mathrm{eV}$. The peaks at $366 \mathrm{eV}$ and $737 \mathrm{eV}$ proved the existing of $\mathrm{Ag}^{+}$in the ANS catalyst ${ }^{57,58}$. Consequently, the discovery of these peaks further confirmed that $\mathrm{Ag}$ atoms were successfully doped into $\mathrm{NaSbO}_{3}$ molecules. Figure 8d depicted the high-resolution XPS spectra of Na2p and Na2s. It was seen that the spectra of $\mathrm{Na} 2 \mathrm{p}$ and $\mathrm{Na} 2 \mathrm{~s}$ could be observed at $33 \mathrm{eV}$ and $52 \mathrm{eV}$, respectively. In conclusion, XPS spectra results further demonstrated the existence elements and their existing forms in ANS catalyst.
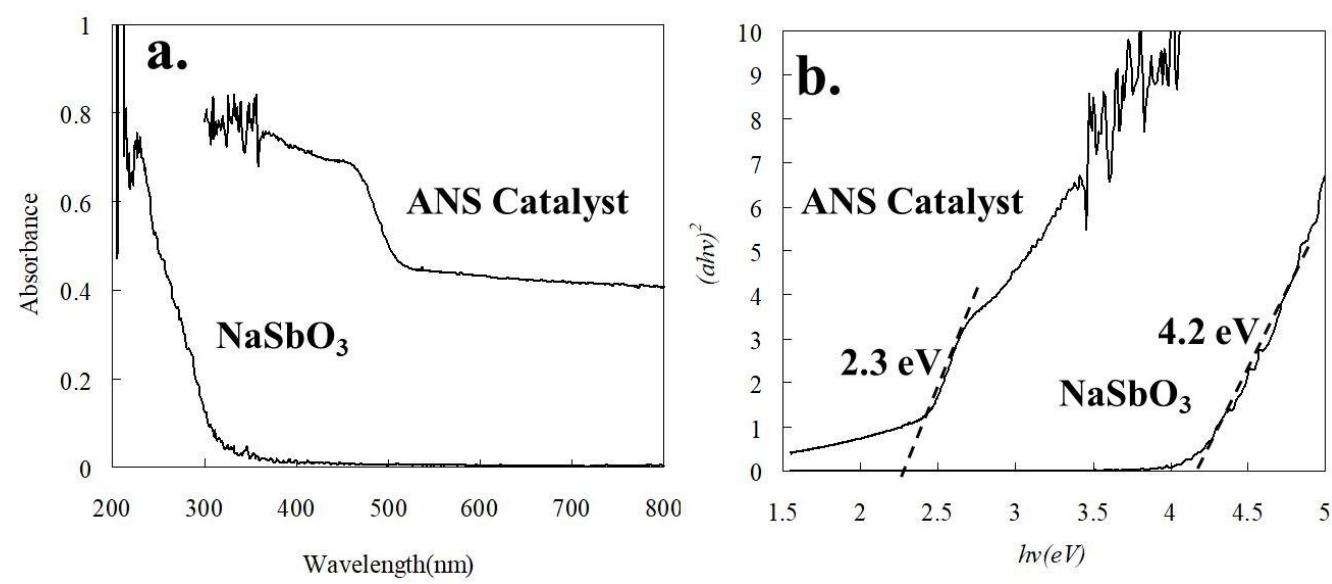

Figure 6. UV-vis diffuse reflectance spectra of $\mathrm{NaSbO}_{3}$ and ANS Catalyst. 


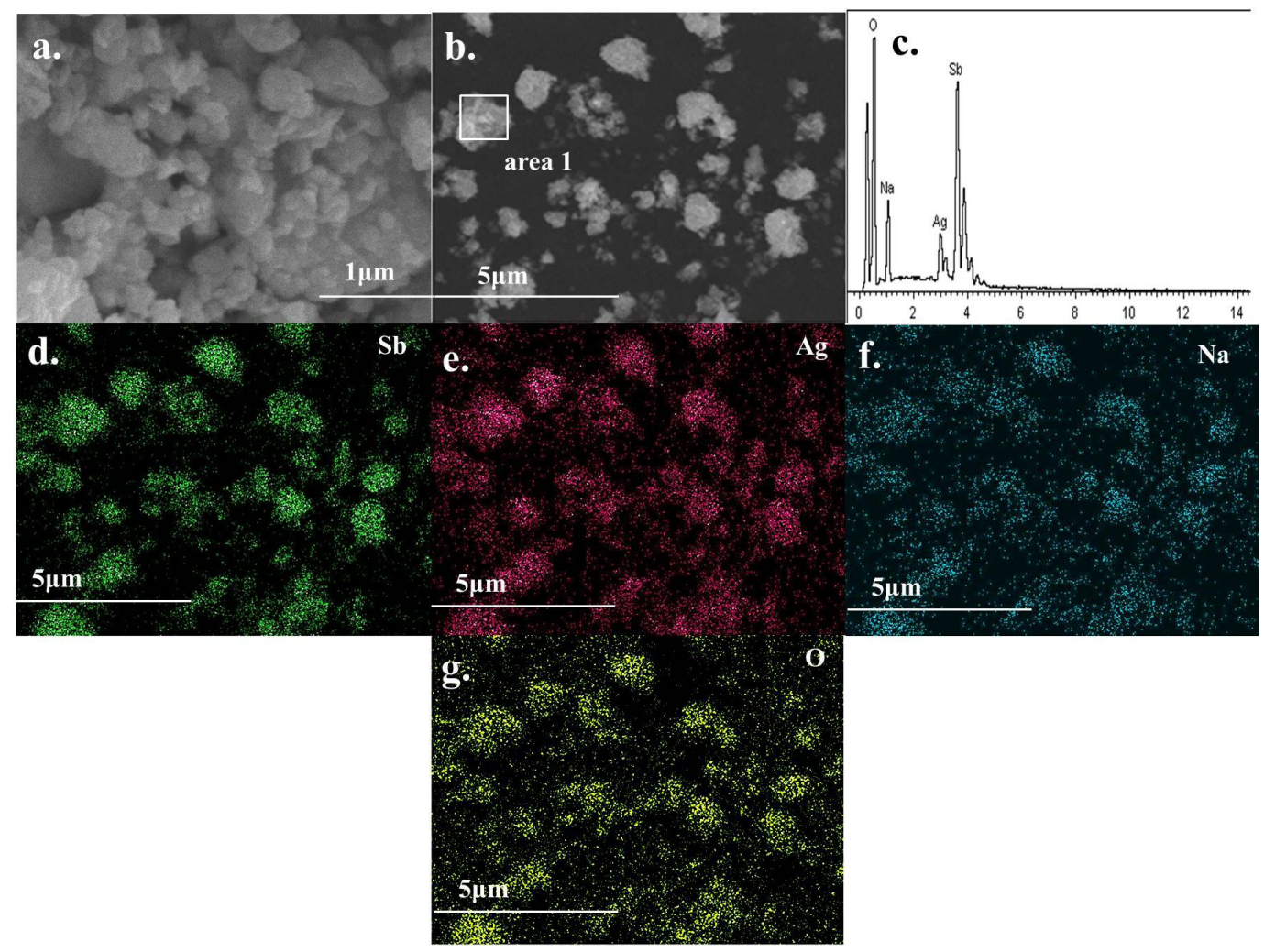

Figure 7. The SEM and EDX results of ANS Catalyst (a. b and c), and the EDX element mapping of Sb (d), Ag (e), Na (f) and O (g).
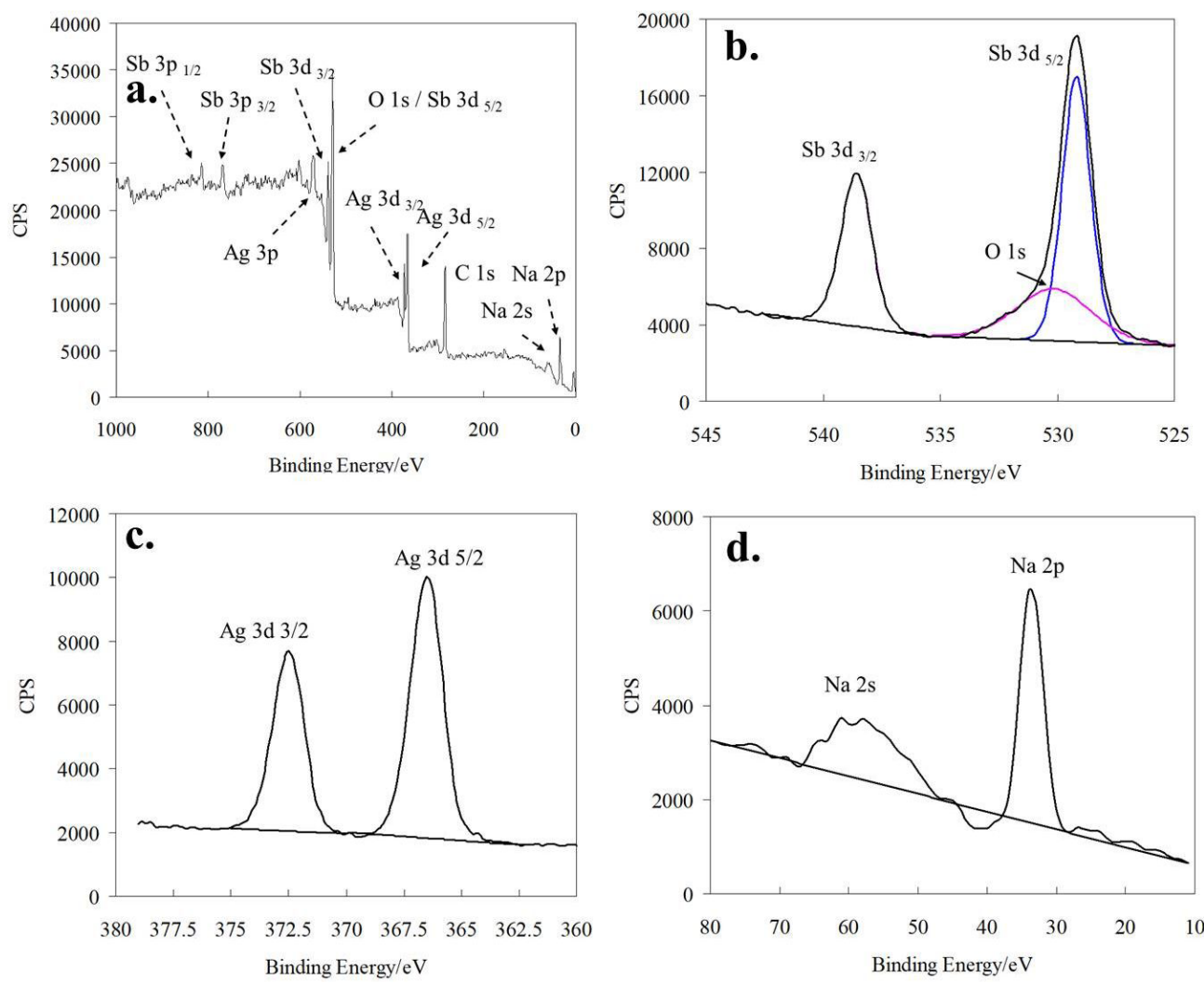

Figure 8. The wide scan XPS spectra (a) of ANS Catalyst; The high-resolution XPS spectra of (b) O1s and Sb 3d, (c) Ag3d, (d) Na2p and Na2s. 


\subsection{Photocatalytic activity}

The photocatalytic activity of ANS catalyst was evaluated through the photocatalytic degradation of MB under visible light in solution, as shown as Figure 9. We investigated the effect of catalyst dosages on the photocatalytic degradation efficiencies of MB. Meanwhile, the degradation efficiencies of $\mathrm{NaSbO}_{3}$ and ANS without light on $\mathrm{MB}$ were also studied as comparisons, respectively. In the degradation process, it was seen from Figure 9a that the photodegradation efficiency of MB increased gradually with the increase of catalyst dosages under visible light. When the catalyst dosage was $0.67 \mathrm{~g} / \mathrm{L}$ at $10 \mathrm{~min}$, the degradation residual rate of MB achieved at $24 \%$. As an efficient photocatalyst, such photodegradation efficiency could be acceptable. In addition, from the comparison of photocatalytic effect (Figure 9b), the addition of photocatalyst greatly promoted the degradation of $\mathrm{MB}$, and the degradation rates for $\mathrm{MB}$ were better than that of other two systems. For instance, the residual rate of MB was approximately $11.1 \%$ just at $30 \mathrm{~min}$ after adding ANS catalyst, while the residual rate were $74.3 \%$ at $30 \mathrm{~min}$ with $\mathrm{NaSbO}_{3}$. However, the catalyst degradation efficiency of MB without illumination was significantly lower than that of the normal photocatalytic system, and the residual rate of MB was still $94.8 \%$ at $30 \mathrm{~min}$. These findings demonstrated that the doping of $\mathrm{Ag}$ atom greatly promoted the photocatalytic activity of $\mathrm{NaSbO}_{3}$ material. The varieties of UV-visible absorption spectra of $\mathrm{MB}$ at different reaction time were depicted in Figure 7f. It suggested that peaks of MB gradually decreased with the reaction time, which stated that MB displayed favorable photodegradation adding ANS catalyst. The photocatalytic performance of ANS catalyst is almost the similar as that of other latest photocatalytic materials $\left(\mathrm{Nd}^{3+}\right.$ doped $\mathrm{BaAl}_{2} \mathrm{O}_{4}^{59}$, cobalt substituted zinc ferrite ${ }^{60}, \mathrm{~N}$ self-doped $\mathrm{TiO}_{2} / \mathrm{ZrO}_{2}$ composite $^{61}, \mathrm{Ag} @ \mathrm{CdSe} /$ Zeoilte nanocomposite ${ }^{62}, \mathrm{CQDs} / \mathrm{CeO}_{2} / \mathrm{SrFe}_{12} \mathrm{O}_{19}{ }^{63}$ ). Besides, we investigated the apparent degradation kinetics via firstorder reaction model, and the equation of which is as follows. The reaction constant $\mathrm{k}\left(\mathrm{min}^{-1}\right)$ and half life of the reactions ( $\mathrm{min}$ ) can be obtained by fitting the experimental results via the Equations 2 and 3.

$\operatorname{Ln}\left(\frac{C}{C_{0}}\right)=-k t$

$T_{\text {half }- \text { life }}=\frac{\operatorname{Ln}(2)}{k}$

Where, $\mathrm{C}_{0}$ represents the initial concentration of $\mathrm{MB}$, and $\mathrm{C}_{\mathrm{t}}$ represents the concentration of $\mathrm{MB}$ at time $\mathrm{t}$

Figure $9 \mathrm{c}$ presented the fitting results of apparent kinetics utilizing the Equation 2 (based on the data in Figure 9b). The $\mathrm{R}^{2}$ values fitted by the equation were $0.9983,0.99519$, and 0.9651 , respectively. They were all above 0.96 , which indicated that the apparent photocatalytic degradation process of MB could be satisfactorily fitted by first-order reaction model. Additionally, the $\mathrm{k}$ value of ANS photogradation system from Figure $7 \mathrm{~d}$ was 0.0823 , which was the largest in three systems. At the same time, the addition of ANS Catalyst greatly reduced the half-life of the reaction (Figure 9e). The half-life of ANS reaction system was about $8.42 \mathrm{~min}$, and that of the other two systems were about 383 min (no-light system) and $86 \mathrm{~min}$ ( $\mathrm{NaSbO} 3$ system), respectively.
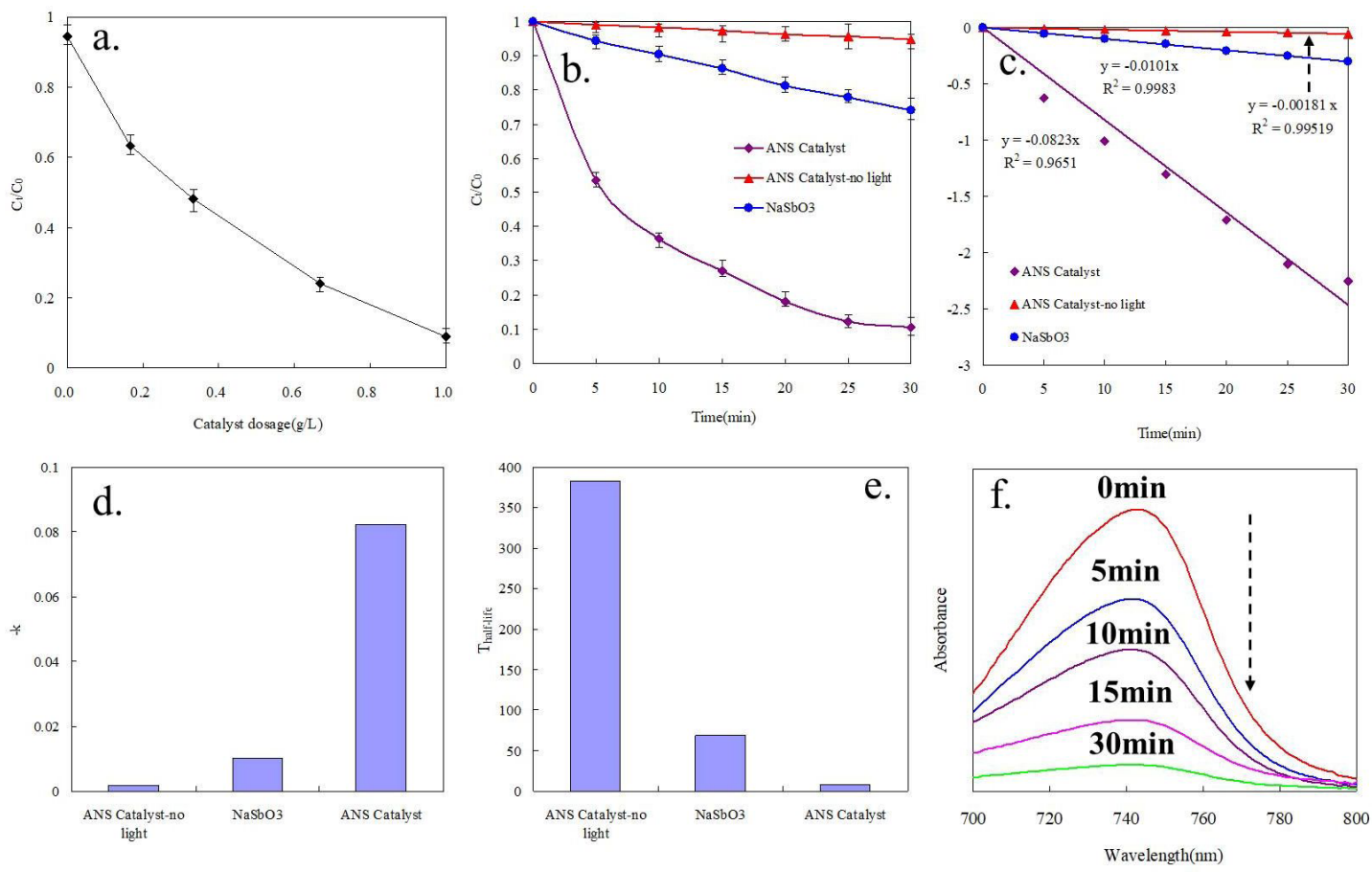

Figure 9. (a) The effect of ANS Catalyst dosage on the removal rate of MB; (b) The photodegradation effect of ANS Catalyst under visible light, $\mathrm{NaSbO}_{3}$, and ANS Catalyst with no light; (c) the apparent fitting findings using the pseudo-first-order model; (d) the constants of first-order reactions for MB photodegradation; (e) The half life of photocatalysis reactions; (f) UV-visible absorption spectra of MB solution degraded by ANS Catalyst. 
Furthermore, we measured the photocatalytic performance of recycled ANS through identifying the photodegradation efficiencies of MB under visible light. The morphology and XRD patterns of reused ANS after three times were also analyzed, as shown as Figure 10. It was seen that, the catalyst maintained amorphous state in comparison of the original sample after the consecutive three times of photocatalysis experiment (Figure 10a). Moreover, it was no obvious variations in the overall appearance of ANS particles. From XRD patterns of ANS after 3rd photocatalysis experiment (Figure 10b), we found that the main peaks could also be appeared contrast with the previous results. Additionally, according to the reuse photodegradation curves of ANS (Figure 10c), there was only a slight reduction of photocatalytic activities after three cycles of recycle, which verified that ANS possessed remarkable photocatalytic stability, and the ANS catalyst could be efficiently reused.

\subsection{Photodegradation mechanism}

The transient photocurrent response of ANS catalyst and $\mathrm{NaSbO}_{3}$ under Xe lamp irradiation was described in Figure 11a. It was seen that $\mathrm{NaSbO}_{3}$ only appeared slight response due to its bigger band gaps energy $(4.5 \mathrm{eV})$, which made $\mathrm{NaSbO}_{3}$ unable to enough excite electrons and holes out under visible light. On the contrary, the photocurrent intensity of ANS catalyst enhanced greatly after the doping of Ag atoms, confirming that ANS catalyst possessed stronger ability of generating and transferring electrons and holes under visible light.
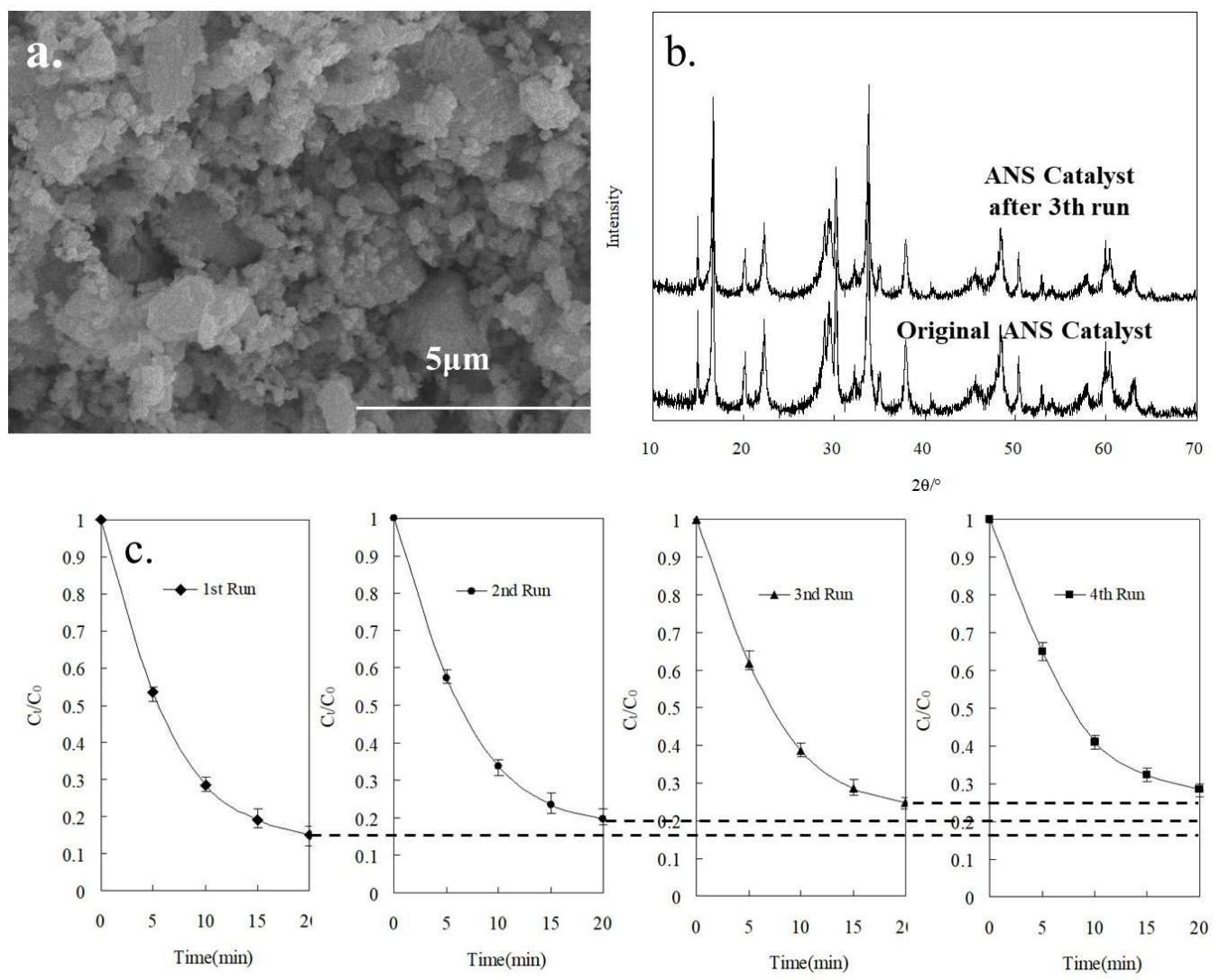

Figure 10. (a) The morphology of catalyst after 3rd photocatalysis experiment ; (b) The XRD patterns of ANS Catalyst before and after 3rd photocatalysis experiment; (c) the degradation curve of ANS catalyst reuse under visible light

Figure $11 \mathrm{~b}$ depicted the EIS value of ANS catalyst and $\mathrm{NaSbO}_{3}$. The radius values of $\mathrm{NaSbO}_{3}$ were bigger than that of ANS, stating that the doped Ag atoms reduced the resistance of charge transfer, thus effectively promoted the reaction process of photocatalysis. In addition, the PL emission spectrum was further conducted to evaluate the combination and separation of photogenerated electrons and holes, as shown in Figure 11c. It was observed that the PL emission spectrum of ANS displayed significant red shift compared with $\mathrm{NaSbO}_{3}$. This stated that the photoluminescence energy of catalyst was lower than that of $\mathrm{NaSbO}_{3}$, and that might be ascribed to the smaller band energy of ANS catalyst. Also, the photoluminescence spectrum intensity of ANS Catalyst did not become stronger than that of $\mathrm{NaSbO}_{3}$, which suggested the $\mathrm{Ag}$ atoms doped could not enhance the combination probability of photogenerated electrons and holes ${ }^{42,43}$. Figure 11d showed the Mott-Schottky measurements of ANS Catalyst. The displayed negative slopes indicated that ANS Catalyst was p-type semiconductors ${ }^{58}$. In addition, the flat-band potential $\left(\mathrm{V}_{\mathrm{fb}}\right)$ could be obtained by M-S curves, corresponding to the intercepts of the $\mathrm{X}$ axis ${ }^{61}$. The $\mathrm{V}_{\mathrm{fb}}$ of ANS Catalyst was $2.44 \mathrm{~V}$ (vs NHE). Because the valence band $(\mathrm{VB})$ potential $\left(\mathrm{E}_{\mathrm{VB}}\right)$ of p-type semiconductors is $0.2 \mathrm{~V}$ positive than $\mathrm{V}_{\mathrm{fb}}$ in theory ${ }^{61}$, the $\mathrm{E}_{\mathrm{VB}}$ of ANS Catalyst should be around $2.64 \mathrm{~V}$. Combined with previous UV-vis analysis, the range between $\mathrm{E}_{\mathrm{CB}}$ (conduction band potential) and $\mathrm{E}_{\mathrm{VB}}$ should be $0.34 \mathrm{~V}-2.64 \mathrm{~V}$. After unit conversion, the range should be $-4.84 \mathrm{eV}$ to $-7.14 \mathrm{ev}$. . 

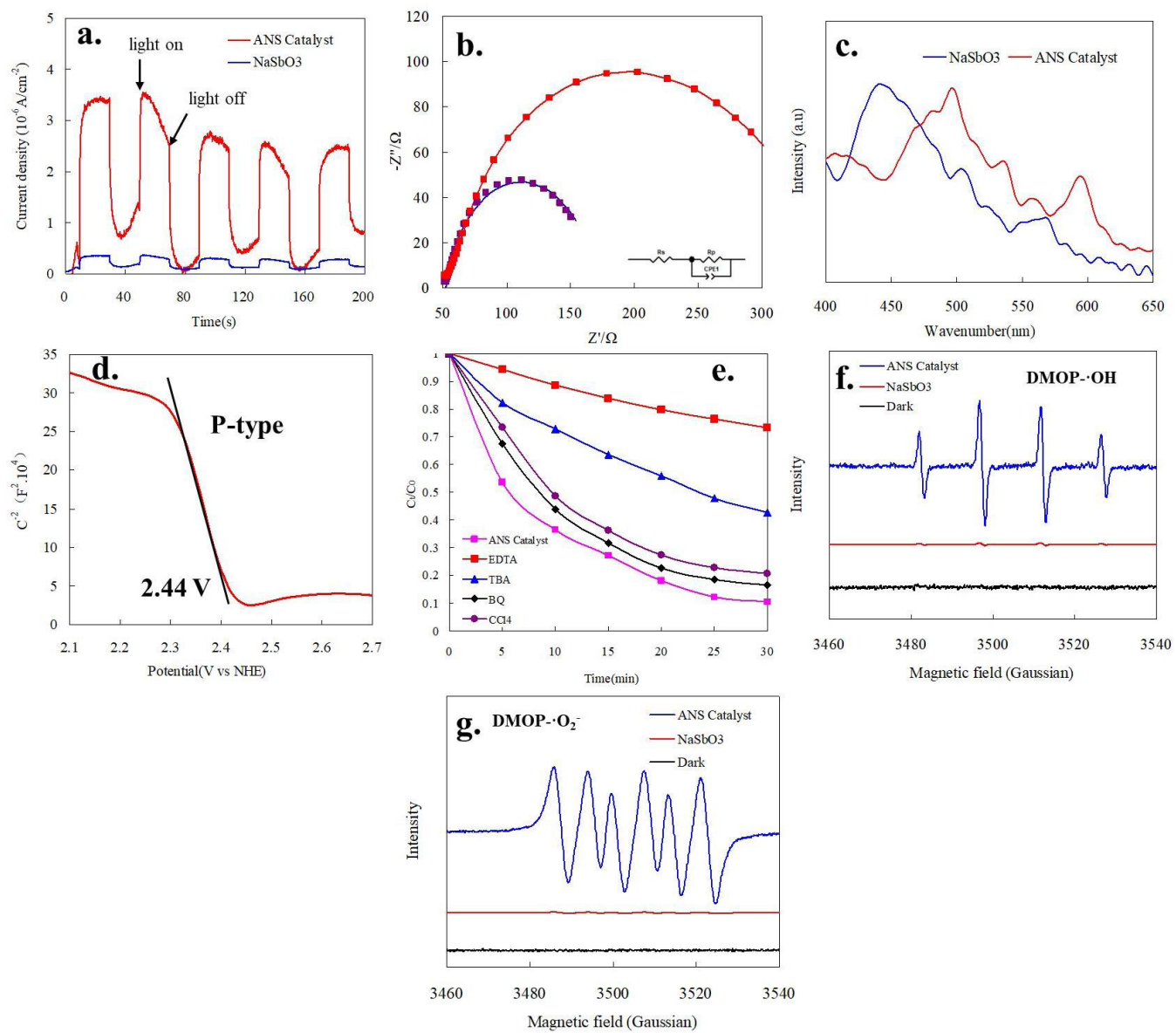

Figure 11. (a) Transient photocurrent responses of ANS Catalyst and $\mathrm{NaSbO}_{3}$ under visible-light irradiation; (b) Electrochemical impedance spectra of ANS Catalyst and $\mathrm{NaSbO}_{3}$; (c) Steady-state PL spectra of ANS Catalyst and $\mathrm{NaSbO}_{3}$; (d) Mott-Schottky curves of ANS Catalyst; (e) photodegradation efficiency by addition of trapping agents; (f) ESR spectra of radical adducts trapped by DMPO in the dark and under visible light irradiation for DMPO-·OH; (g) ESR spectra of radical adducts trapped by DMPO in the dark and under visible light irradiation for $\mathrm{DMPO}-\mathrm{O}_{2}$ :

It is known that, the photocatalytic degradation process of organic pollutants mainly depends on the strong redox of free electrons, holes and related free radicals released by photocatalysts. To evaluate which species playing active roles in the process of photocalytic degradation reaction, we took advantage of trapping agents putting into the reaction mixtures. On the basis of references ${ }^{66,67}$, EDTA-2Na could be used as a hole trapper, TBA as a hydroxyl radical trapper, $\mathrm{BQ}$ as an oxygen free radical trapper, and $\mathrm{CCl}_{4}$ as an electron trapper. Figure 11e described the effect of different trapping agents on the photocatalytic activity of MB. It displayed that the photocatalytic efficiencies of ANS catalyst were all decreased after adding trapping agents. This phenomenon confirmed that the photocatalytic activities were inhibited with the coexistent of these scavengers, and photocatalytic degradation path from Equation 4-6 was probably happen during the degradation process.

$\mathrm{O}_{2}+e^{-} \rightarrow \cdot \mathrm{O}_{2}^{-}$

$\cdot \mathrm{O}_{2}^{-}+\mathrm{MB} \rightarrow$ Degradation - products
$\mathrm{H}_{2} \mathrm{O} / \mathrm{OH}^{-}+\mathrm{h}^{+} \rightarrow \cdot \mathrm{OH}^{-}$

\section{$\cdot \mathrm{OH}+\mathrm{MB} \rightarrow$ Degradation - products}

Furthermore, ESR spin-trapping spectra were measured using the DMPO technique to detect the hydroxyl radicals (DMPO-・OH) and the superoxide radicals (DMPO- $\cdot \mathrm{O}_{2}^{-}$), to prove the action mechanism of active radicals in photocatalytic reaction, as shown as Figure 11f and Figure 11g. In both $\mathrm{NaSbO}_{3}$ and ANS catalyst absence of light system, there was no characteristic peaks of $\cdot \mathrm{OH}$ or ${ }^{-} \mathrm{O}_{2}^{-}$were measured. That indicated in both $\mathrm{NaSbO}_{3}$ and ANS lack of light system, there were no excited electrons and holes. Accordingly, $\mathrm{OH}$ or $\mathrm{O}_{2}^{-}$would not be appeared. In contrast, the typical peaks of $\bullet \mathrm{OH}$ with the intensity ratio of $1: 2: 2: 1$ and that of $\bullet \mathrm{O}_{2}$ were detected under visible light irradiation ${ }^{68,69}$, which demonstrated that both $\bullet \mathrm{OH}$ and $\bullet_{2}^{-}$were produced in the process of ANS photocatalytic degradation. Moreover, these findings were in line with that of Figure 11f. The action process of these free radicals might be one of the main photocatalytic mechanisms for pollutant degradation. Besides, among these scavengers, EDTA-2Na and TBA exhibited more obvious influences on the photocatalytic 
reaction process. Additionally, these findings implied that the capture of holes and hydroxyl radicals was likely to seriously lower the photodegradation activity, which might be explained by the main degradation path of MB as Equation 5 and 6 under ANS photocatalytic system.

To further clarify the photocatalytic mechanism of ANS catalyst, the VASP software was utilized to investigate the DFT calculation for $\mathrm{NaSbO}_{3}$ and ANS catalyst. The structure of the material was optimized theoretically, and the optimized structures of $\mathrm{NaSbO}_{3}$ and ANS catalyst were shown in Figure $3 \mathrm{a}$. In the process of constructing the crystal structure of ANS catalyst, a $\mathrm{Na}$ atom in the original $\mathrm{NaSbO}_{3}$ crystal was replaced by an $\mathrm{Ag}$ atom, as shown as Figure $2 \mathrm{~b}$, and the lattice parameters before and after doping was shown in Table 1. It was observed that the crystal parameters of $\mathrm{NaSbO}_{3}$ and ANS Catalyst calculated theoretically were different from the experimental results. However, the theoretical calculations also indicated that the unit cell parameters of ANS Catalyst were smaller than that of $\mathrm{NaSbO}_{3}$ through the doping of $\mathrm{Ag}$, which was consistent with the results obtained by the previous XRD analysis. By comparing Figure 3 a and Figure 3b, it could be seen that a $\mathrm{Na}$ atom in the crystal structure of $\mathrm{NasbO}_{3}$ was replaced by an $\mathrm{Ag}$ atom, and its atomic coordinates changed from $(0.667,0.333,0.975)$ to $(0.667,0.333,0.929)$. According to the calculation results of atomic proportion in the theory model (Figure $3 b$ ), the proportion of doped $\mathrm{Ag}$ is about $3.3 \%$. The proportion of Ag element in EDX and XPS analysis results is $2.47 \%$ and $3.86 \%$, respectively. Considering the difference between experiment data and theoretical analysis, the theoretical model of ANS could be

Table 1. The lattice parameters before and after doping (DFT calculation).

\begin{tabular}{ccc}
\hline & Before doping/Am & After doping/Åm \\
\hline $\mathrm{a}$ & 4.96950 & 4.91614 \\
\hline $\mathrm{b}$ & 4.96950 & 4.91614 \\
\hline $\mathrm{c}$ & 14.00391 & 12.88423 \\
\hline
\end{tabular}

a.

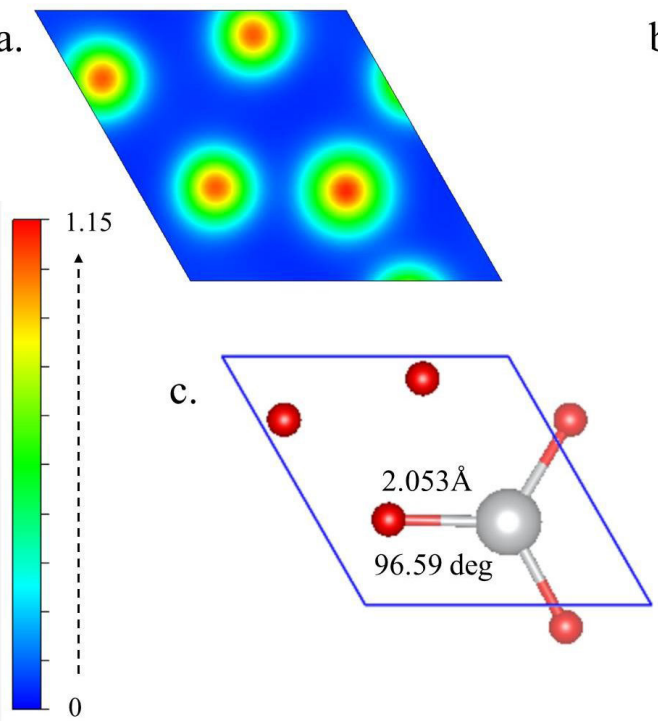

acceptable. The lattice parameters of the crystal structure had also changed (Table 1).

On the basis of structure optimization, we calculated the charge density of $\mathrm{NaSbO}_{3}$ and ANS catalyst, as shown as Figure 12 (Charge density distribution). The charge was mainly distributed around the atomic position, which indicated that bonds of Ag-O and $\mathrm{Na}-\mathrm{O}$ in the crystal structure were both typical ionic bonds. The length of Ag-O bond was $2.053 \AA$, and the bond angle was $96.59 \mathrm{deg}$ (Figure 12c). While the length of Na-O bond was $2.038 \AA$, and the bond angle was $103.2 \mathrm{deg}$ (Figure 12d). In addition, we observed the charge of Ag atoms in Figure 12a. This phenomenon indicated that $\mathrm{Ag}$ atom doping into $\mathrm{NaSbO}_{3}$ was extremely stable presented in the crystal. Moreover, the charge of $\mathrm{Na}$ atom could not be observed in Figure 12b, which might due to the fact that $\mathrm{Na}$ atom was far away from the charge density center.

Figure 13 displayed the DOS calculation results of $\mathrm{NaSbO}_{3}$ and ANS catalyst. According to the computation findings (Figure 13a and Figure 13b), the band gaps of $\mathrm{NaSbO}_{3}$ and ANS catalyst were $4.15 \mathrm{eV}$ and $2.05 \mathrm{eV}$, respectively, which was close to that of the analytical results of diffuse reflection. By comparing Figure 13a with Figure 13b, it was seen that the reduction in the band gap of ANS catalyst was due to the appearance of some new state densities near Fermi energy level of original $\mathrm{NaSbO}_{3}$, which was mainly contributed by the d orbital of Ag atoms doped in the crystals. Similar results could also be obtained from the energy band calculation results in Figure 14. We observed that $\mathrm{NaSbO}_{3}$ was a typical direct band gap semiconductor (Figure 14a), and many additional bands appeared near Fermi energy level of original $\mathrm{NaSbO}_{3}$ by doping Ag atoms. The addition of these energy bands greatly reduced the band gap of ANS catalyst to $2.05 \mathrm{eV}$. Hence, theoretically, the doping of $\mathrm{Ag}$ atoms provided a suitable additional band, which made ANS catalyst exhibit remarkable visible light absorption performances.

Figure 15 depicted the theoretical calculation results of optical properties for $\mathrm{NaSbO}_{3}$ and ANS catalyst. The

b.

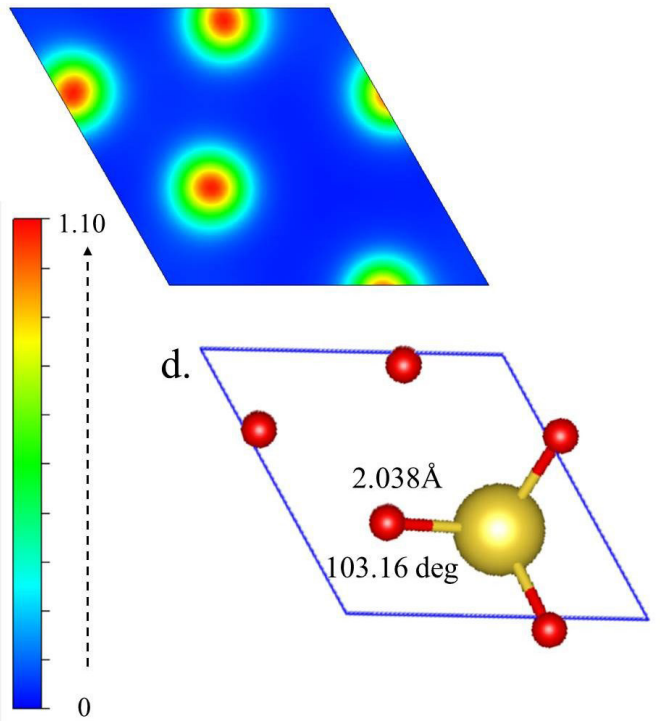

Figure 12. Charge density distribution ( 001 surface, distance from origin is $0.1 \mathrm{~d}$ ) of ANS Catalyst (a) and $\mathrm{NaSbO}_{3}(\mathrm{~b})$. 

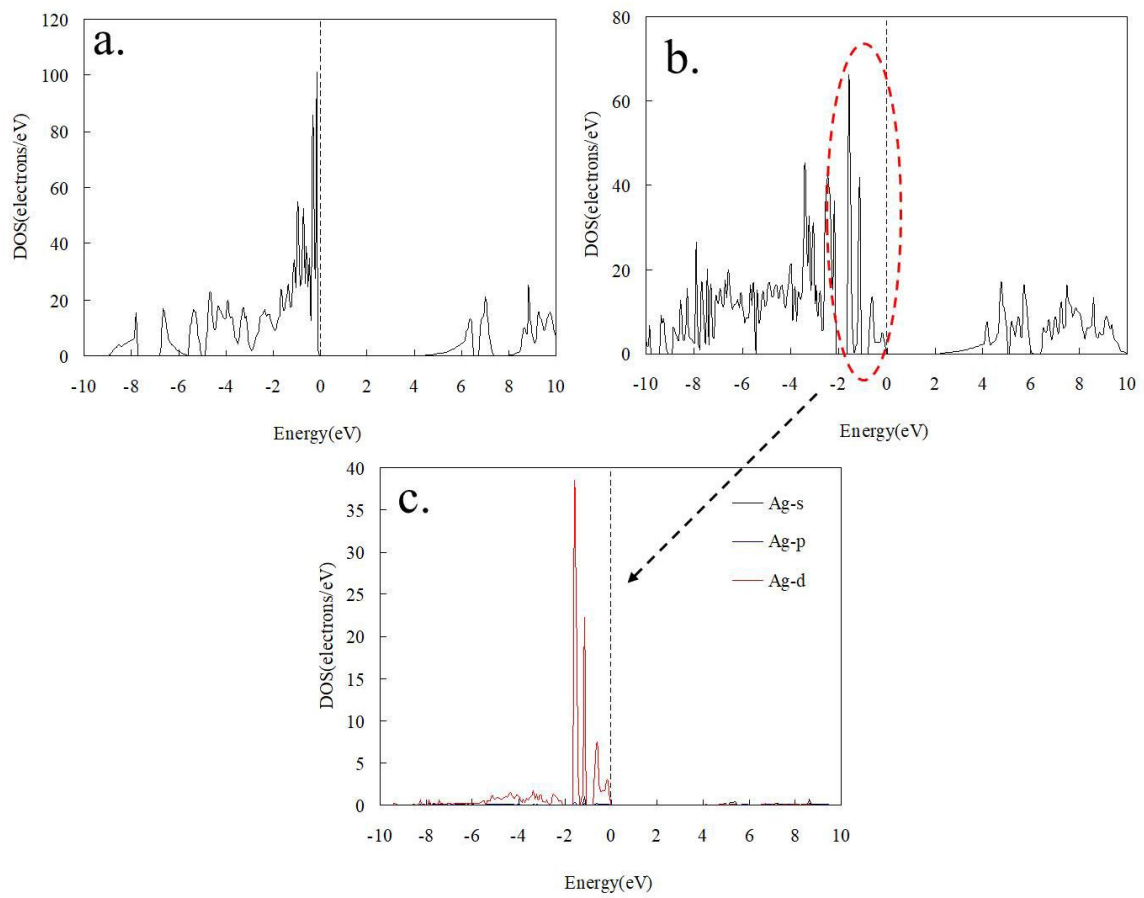

Figure 13. Density of States of $\mathrm{NaSbO}_{3}$ (a), ANS Catalyst (b) and pDOS of Ag atom in ANS Catalyst (c).

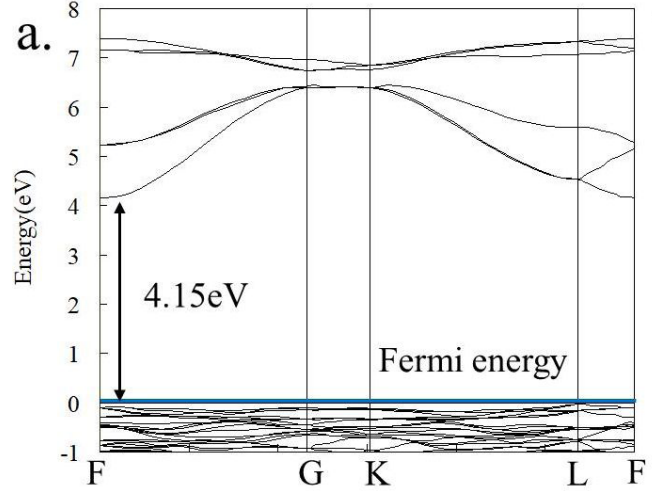

Figure 14. The band structure of $\mathrm{NaSbO}_{3}$ (a), ANS Catalyst (b).

overall optical properties of $\mathrm{NaSbO}_{3}$ and ANS catalyst were relatively similar (Figure 15a, c). However, through comparing Figure 15b and Figure 15d, it was seen that the visible light absorption of ANS catalyst was significantly higher than that of $\mathrm{NaSbO}_{3}$ under visible light ranged from $1 \mathrm{eV}$ to $3 \mathrm{eV}$. Especially in xy direction of crystal, the maximum visible light absorption coefficient of $\mathrm{NaSbO}_{3}$ crystal was $0.06\left(10^{5} \mathrm{~cm}^{-1}\right)$, while the visible light coefficient curve of ANS catalystl was significantly higher, and the maximum value was $0.16\left(10^{5} \mathrm{~cm}^{-1}\right)$. These findings confirmed that the doping of $\mathrm{Ag}$ ion effectively improved the visible light absorption of material, which was very beneficial to the improvement of photocatalytic performance.

Figure 16 displayed the calculation results of work function for $\mathrm{NaSbO}_{3}$ and $\mathrm{ANS}$ catalyst. The work function of $\mathrm{NaSbO}_{3}$ and ANS catalyst were $4.63 \mathrm{eV}$ and $6.72 \mathrm{eV}$, respectively. The

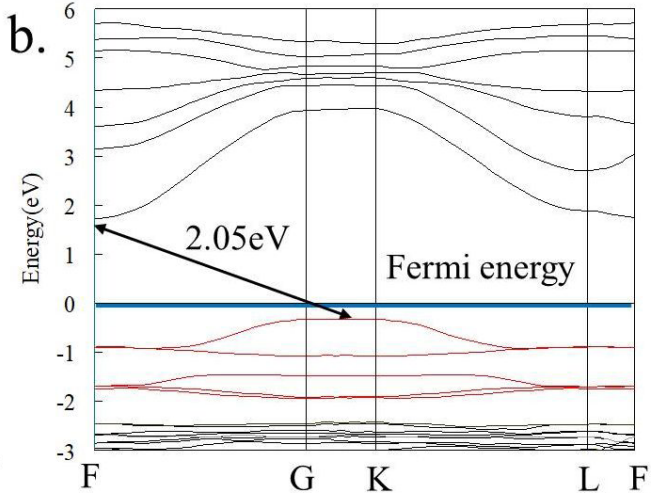

calculation results of the range between $\mathrm{E}_{\mathrm{CB}}$ and $\mathrm{E}_{\mathrm{VB}}$ was basically consistent with the previous Mott-Schottky curves results. This phenomenon indicated that the doping of Ag atoms not only reduced the band gap of material, but also improved the work function. By reducing the band gap, the catalyst displayed obvious visible light excitation performance. Moreover, the improvement of work function enhanced the oxidation ability of photogenerated holes, and more hydroxyl radicals were formed in system, making the oxidation of photogenerated holes and the reaction of hydroxyl radicals became the main mechanism for degrading pollutants. These findings were in line with previous photocatalytic results in hydroxyl radical and photogenerated hole trapping agent systems. The schematic diagram for the whole photocatalytic degradation mechanism by $\mathrm{Ag}$ doped $\mathrm{NaSbO}_{3}$ in this reaction was described in Figure 17. 

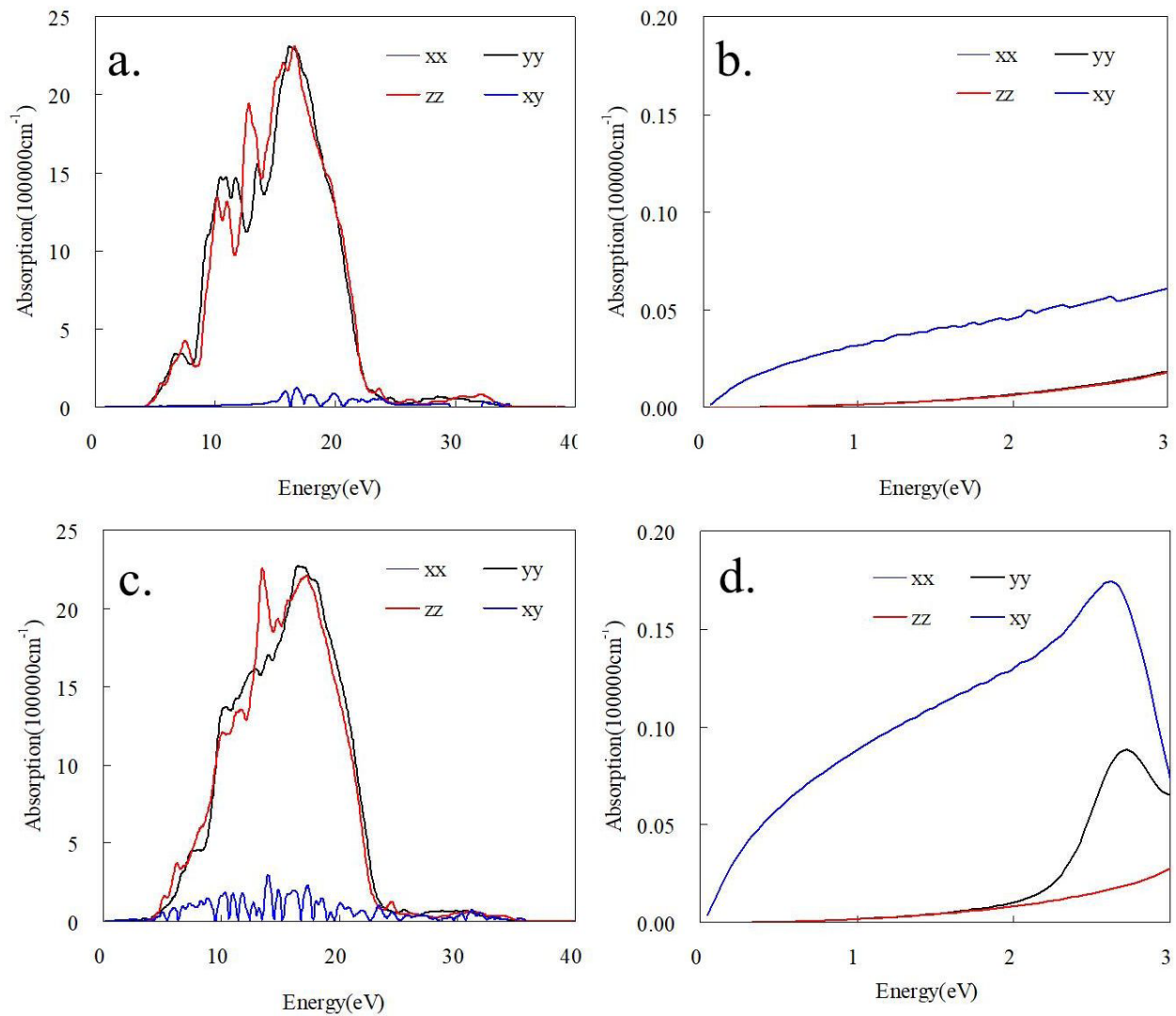

Figure 15. The calculation optical properties of $\mathrm{NaSbO}_{3}$ (a), $\mathrm{NaSbO}_{3}$ under visible light (b), ANS Catalyst (c), ANS Catalyst under visible light (d).
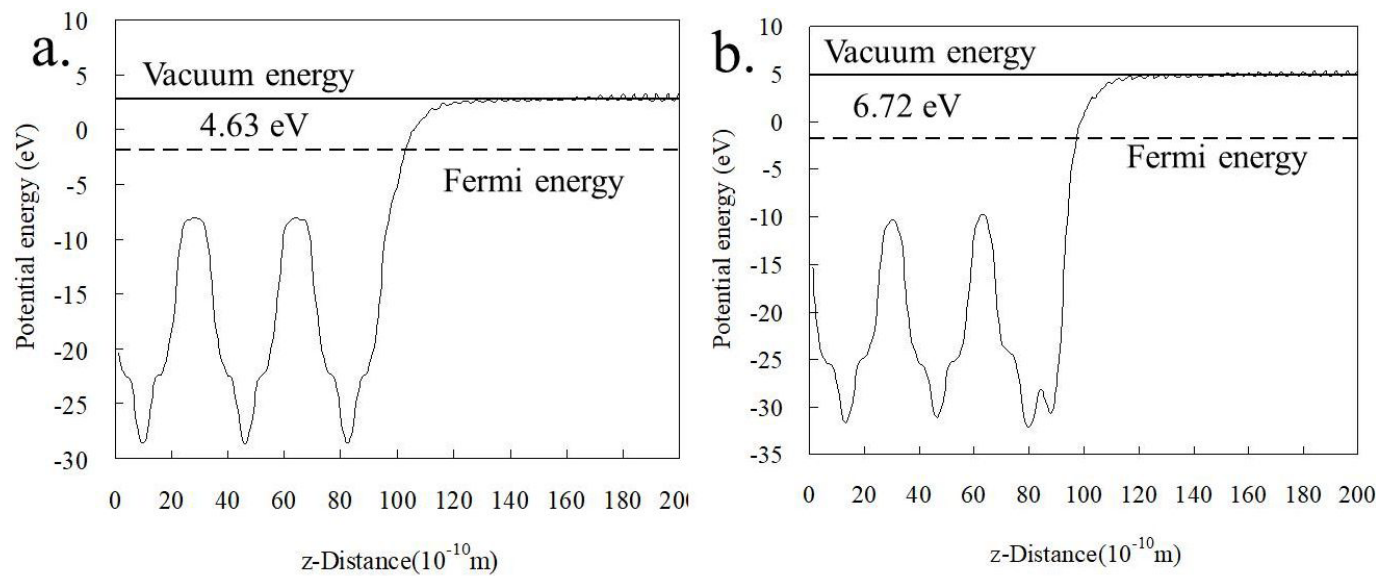

c.

Vacuum energy

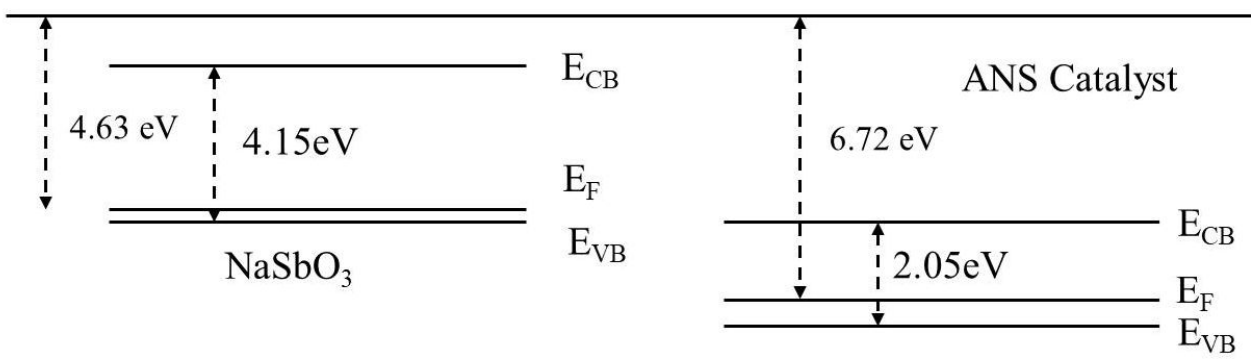

Figure 16. Work function of $\mathrm{NaSbO}_{3}$ (a) and ANS Catalyst (b), Comparison of energy band structures of $\mathrm{NaSbO}_{3}$ and ANS Catalyst. 


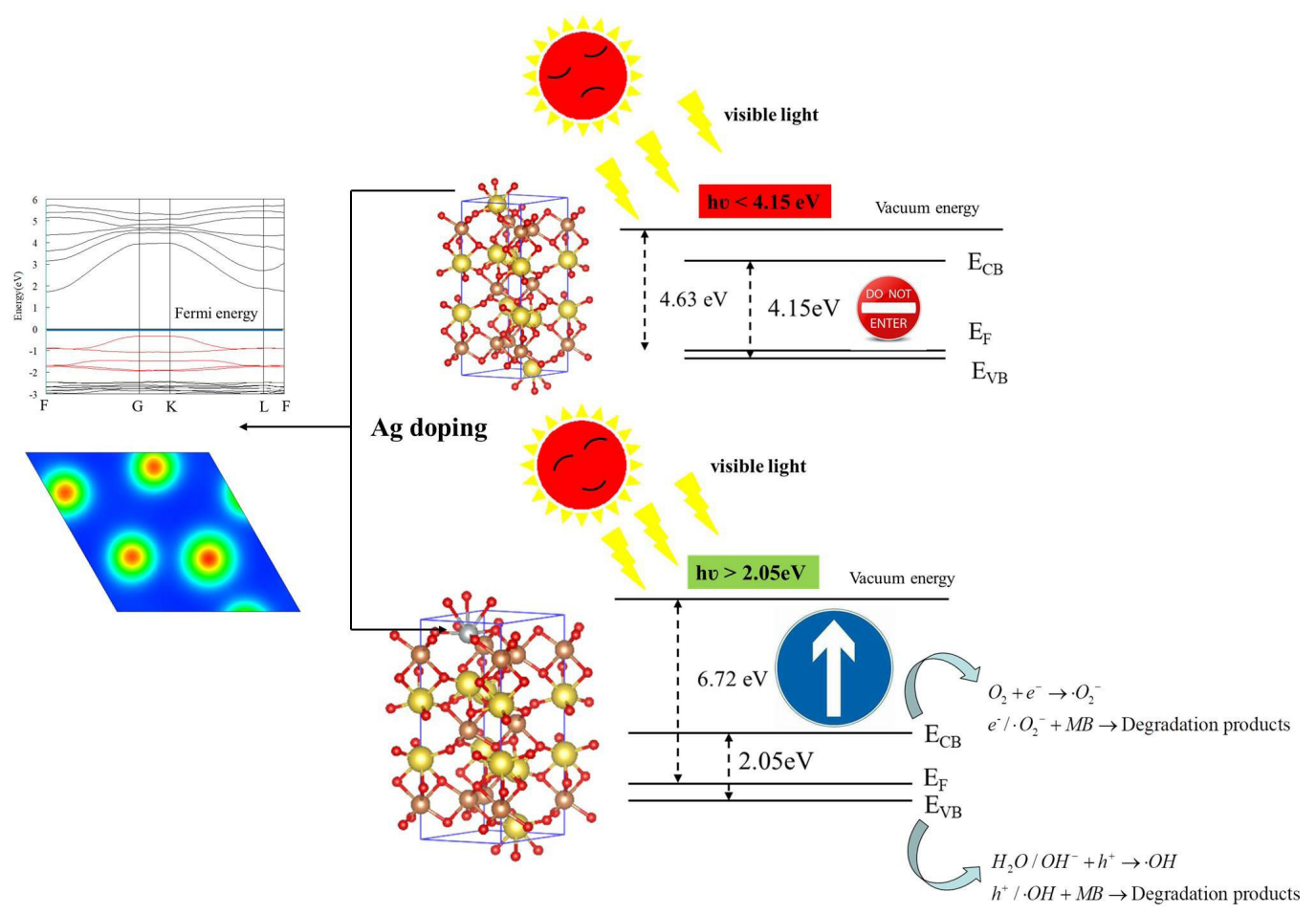

Figure 17. Schematic diagram of photocatalytic degradation by $\mathrm{Ag}$ doped $\mathrm{NaSbO}_{3}$ (ANS catalyst).

\section{Conclusions}

A photocatalyst, ANS catalyst, was prepared, characterized, and applied to the photocatalytic degradation of MB. The properties of ANS catalyst were fully measured by various instruments. The characterization results confirmed that, the doping of $\mathrm{Ag}$ atoms into $\mathrm{NaSbO}_{3}$ crystals reduced the band gap, and ANS catalyst exhibited favorable absorption performance of visible light. The photodegradation experiments stated that ANS catalyst effectively enhanced the photocatalytic degradation of MB under visible light, and the first-order reaction model could perfectly fit the process of photodegradation. In addition, ANS catalyst displayed excellent photocatalytic performance after consecutive three times of recycle. The electrochemical experiments findings revealed that ANS catalyst had a strong ability to generate and transfer electrons and holes under visible light. $\mathrm{Ag}$ atoms doping into $\mathrm{NaSbO}_{3}$ decreased the resistance of charge transfer. Accordingly, the photocatalytic degradation of MB was greatly enhanced. Besides, The active species of $\bullet \mathrm{OH}$ and $\bullet \mathrm{O}_{2}$ - could be detected in the photodegradation process, and they were vital to the photocatalytic reaction. DFT computation illustrated that, by doping with Ag atoms, many additional energy bands appeared near Fermi energy level of $\mathrm{NaSbO}_{3}$, which greatly reduced the band gap of ANS catalyst to $2.05 \mathrm{eV}$, and thus favored to effective absorb visible light. Additionally, the workfunction was improved during the photocatalytic process, which could increase the oxidation ability of photogenerated holes, and form more hydroxyl radicals, and then accelerate the photocatalytic degradation of $\mathrm{MB}$.

\section{Acknowledgements}

This work is supported by Science Foundation of Jiangsu Colleges and Universities (Grant No. 17KJD610001, 17KJD610002).

\section{References}

1. Rout PR, Zhang TC, Bhunia P, Surampalli RY. Treatment technologies for emerging contaminants in wastewater treatment plants: a review. Sci Total Environ. 2021;753:141990.

2. Ben W, Zhu B, Yuan X, Zhang Y, Yang M, Qiang Z. Occurrence, removal and risk of organic micropollutants in wastewater treatment plants across China: comparison of wastewater treatment processes. Water Res. 2018;130:38-46.

3. Ahmed SF, Mofijur M, Nuzhat S, Chowdhury AT, Rafa N, Uddin MA, et al. Recent developments in physical, biological, chemical, and hybrid treatment techniques for removing emerging contaminants from wastewater. J Hazard Mater. 2021;416:125912.

4. Sánchez JB, Vuono M, Dionisi D. Model-based comparison of sequencing batch reactors and continuous-flow activated sludge processes for biological wastewater treatment. Comput Chem Eng. 2021;144:107127.

5. Chai WS, Tan WG, Munawaroh HSH, Gupta VK, Show PL. Multifaceted roles of microalgae in the application of wastewater biotreatment: a review. Environ Pollut. 2021;269:116236.

6. Ai J, Wang Z, Dionysiou DD, Liu M, Deng Y, Tang M, et al. Understanding synergistic mechanisms of ferrous iron activated sulfite oxidation and organic polymer flocculation for enhancing wastewater sludge dewaterability. Water Res. 2021;189:116652.

7. Chen C, Cheng T, Zhang X, Wu R, Wang Q. Synthesis of an Efficient $\mathrm{Pb}$ Adsorption Nano-crystal under strong alkali hydrothermal environment using a gemini surfactant as directing agent. J Chem Soc Pak. 2019;41(6):1034-8. 
8. Zhang X, Cheng T, Chen C, Wang L, Deng Q, Chen G, et al. Synthesis of a novel magnetic nano-zeolite and its application as an efficient heavy metal adsorbent. Mater Res Express. 2020;7(8):085007.

9. Cheng T, Chen C, Tang R, Han C-H, Tian Y. Competitive adsorption of $\mathrm{Cu}, \mathrm{Ni}, \mathrm{Pb}$, and $\mathrm{Cd}$ from aqueous solution onto fly ash-based linde $\mathrm{F}(\mathrm{K})$ zeolite. Iran J Chem Chen Eng. 2018;37(1):61-72.

10. Liu C, Xia J, Gu J, Wang W, Liu Q, Yan L, et al. Multifunctional CNTs-PAA/MIL101(Fe)@Pt composite membrane for highthroughput oily wastewater remediation. J Hazard Mater. 2021;403:123547.

11. Tomczak W, Gryta M. Application of ultrafiltration ceramic membrane for separation of oily wastewater generated by maritime transportation. Separ Purif Tech. 2021;261:118259.

12. Chen C, Cheng T. Wet air oxidation and catalytic wet air oxidation for refinery spent caustics degradation. J Chem Soc Pak. 2013;35(2):243-9.

13. Oliveira AS, Baeza JA, Miera BS, Calvo L, Rodriguez JJ, Gilarranz MA. Aqueous phase reforming coupled to catalytic wet air oxidation for the removal and valorisation of phenolic compounds in wastewater. J Environ Manage. 2020;274:111199.

14. Ma D, Yi H, Lai C, Liu X, Huo X, An Z, et al. Critical review of advanced oxidation processes in organic wastewater treatment. Chemosphere. 2021;275:130104.

15. Amorim SMD, Sapatieri JC, Moritz DE, Domenico MD, Laqua LADC, Moura-Nickel CD, et al. Antifungal and photocatalytic activity of smart paint containing porous microspheres of $\mathrm{TiO} 2$. Mater Res. 2019;22(6):1-8.

16. Zhang D, Wang J. In situ photoactivated plasmonic Ag3PO4@ silver as a stable catalyst with enhanced photocatalytic activity under visible light. Mater Res. 2017;20(3):702-11.

17. Li Y, Ma W. Photocatalytic oxidation technology for indoor air pollutants elimination: a review. Chemosphere. 2021;280:130667.

18. Wang Q, Gao Q, Al-Enizi AM, Nafady A, Ma S. Recent advances in MOF-based photocatalysis: environmental remediation under visible light. Inorg Chem Front. 2020;7(2):300-39.

19. Rueda-Marquez JJ, Levchuk I, Ibanez PF, Sillanpaa M. A critical review on application of photocatalysis for toxicity reduction of real wastewaters. J Clean Prod. 2020;258:120694.

20. Yong X, Schooneb MAA. The absolute energy positions of conduction and valence bands of selected semiconducting minerals. Am Mineral. 2000;85:543-56.

21. Zhang M, Yang Y, An X, Hou L-a. A critical review of g-C3N4based photocatalytic membrane for water purification. Chem Eng J. 2021;412:128663.

22. Wang X, Hu C, An H, Zhu D, Zhong Y, Wang D, et al. Photocatalytic removal of $\mathrm{MB}$ and hydrogen evolution in water by $(\mathrm{Sr} 0.6 \mathrm{Bi} 0.305) 2 \mathrm{Bi} 2 \mathrm{O} 7 / \mathrm{TiO} 2$ heterostructures under visible-light irradiation. Appl Surf Sci. 2021;544:148920.

23. Zhang Y, Zhou J, Feng Q, Chen X, Hu Z. Visible light photocatalytic degradation of MB using UiO-66/g-C3N4 heterojunction nanocatalyst. Chemosphere. 2018;212:523-32.

24. Somraksa W, Suwanboon S, Amornpitoksuk P, Randorn C. Physical and photocatalytic properties of $\mathrm{CeO} 2 / \mathrm{ZnO} / \mathrm{ZnA} 12 \mathrm{O} 4$ ternary nanocomposite prepared by co-precipitation method. Mater Res. 2020;23(1):1-10.

25. Sun L, Shao Q, Zhang Y, Jiang H, Ge S, Lou S, et al. N selfdoped $\mathrm{ZnO}$ derived from microwave hydrothermal synthesized zeolitic imidazolate framework- 8 toward enhanced photocatalytic degradation of methylene blue. J Colloid Interface Sci. 2020;565:142-55.

26. Tang S, Wang Z, Yuan D, Zhang Y, Qi J, Rao Y, et al. Enhanced photocatalytic performance of BiVO4 for degradation of methylene blue under LED visible light irradiation assisted by peroxymonosulfate. Int J Electrochem Sci. 2020;15(3):2470-80.
27. Wang Y, Ding K, Xu R, Yu D, Wang W, Gao P, et al. Fabrication of BiVO4/BiPO4/GO composite photocatalytic material for the visible light-driven degradation. J Clean Prod. 2020;247:119108.

28. Zhang W, Xing P, Zhang J, Chen L, Yang J, Hu X, et al. Facile preparation of novel nickel sulfide modified $\mathrm{KNbO} 3$ heterojunction composite and its enhanced performance in photocatalytic nitrogen fixation. J Colloid Interface Sci. 2021;590:548-60.

29. Qin Y, Li H, Lu J, Meng F, Ma C, Yan Y, et al. Nitrogen-doped hydrogenated $\mathrm{TiO} 2$ modified with $\mathrm{CdS}$ nanorods with enhanced optical absorption, charge separation and photocatalytic hydrogen evolution. Chem Eng J. 2020;384:123275.

30. Mu R, Ao Y, Wu T, Wang C, Wang P. Synergistic effect of molybdenum nitride nanoparticles and nitrogen-doped carbon on enhanced photocatalytic hydrogen evolution performance of CdS nanorods. J Alloys Compd. 2020;812:151990.

31. Chen P, Chen L, Ge S, Zhang W, Wu M, Xing P, et al. Microwave heating preparation of phosphorus doped g-C3N4 and its enhanced performance for photocatalytic $\mathrm{H} 2$ evolution in the help of Ag3PO4 nanoparticles. Int J Hydrogen Energy. 2020;45(28):14354-67.

32. Tang M, Ao Y, Wang C, Wang P. Facile synthesis of dual Z-scheme g-C3N4/Ag3PO4/AgI composite photocatalysts with enhanced performance for the degradation of a typical neonicotinoid pesticide. Appl Catal B. 2020;268:118395.

33. Shao B, Liu X, Liu Z, Zeng G, Liang Q, Liang C, et al. A novel double Z-scheme photocatalyst Ag3PO4/Bi2S3/Bi2O3 with enhanced visible-light photocatalytic performance for antibiotic degradation. Chem Eng J. 2019;368:730-45.

34. He Y, Zeng L, Feng Z, Zhang Q, Zhao X, Ge S, et al. Preparation, characterization, and photocatalytic activity of novel $\mathrm{AgBr}$ / ZIF-8 composites for water purification. Adv Powder Technol. 2020;31(1):439-47.

35. Wang Z, Wang K, Li Y, Jiang L, Zhang G. Novel BiSbO4/ $\mathrm{BiOBr}$ nanoarchitecture with enhanced visible-light driven photocatalytic performance: oxygen-induced pathway of activation and mechanism unveiling. Appl Surf Sci. 2019;498:143850.

36. Wang Z, Jiang L, Wang K, Li Y, Zhang G. Novel AgI/BiSbO4 heterojunction for efficient photocatalytic degradation of organic pollutants under visible light: interfacial electron transfer pathway, DFT calculation and degradation mechanism study. J Hazard Mater. 2021;410:124948.

37. Wang Z, Cheng Q, Wang X, Li J, Li W, Li Y, et al. Carbon dots modified bismuth antimonate for broad spectrum photocatalytic degradation of organic pollutants: boosted charge separation, DFT calculations and mechanism unveiling. Chem Eng J. 2021;418:129460.

38. Chen DP, Bowers W, Skrabalak SE. Aerosol-assisted combustion synthesis of single-crystalline $\mathrm{NaSbO} 3$ nanoplates: a topotactic template for ilmenite AgSbO3. Chem Mater. 2015;27(1):174-80.

39. Ashok J, Brik MG, Kumar VR, Veeraiah N. Energy band structure and optical band gap calculations of $\mathrm{AgSbO} 3$ photocatalystic pyrochlore crystal phase embedded in $\mathrm{Ag} 2 \mathrm{O}$ doped sodium antimonate glass ceramics. Optik. 2020;206:164345.

40. Singh J, Uma S. Efficient photocatalytic degradation of organic compounds by ilmenite $\mathrm{AgSbO} 3$ under visible and UV light irradiation. J Phys Chem C. 2009;113(28):12483-8.

41. Laurita G, Page K, Sleight AW, Subramanian MA. Structural investigation of the substituted pyrochlore $\mathrm{AgSbO} 3$ through total scattering techniques. Inorg Chem. 2013;52(19):11530-7.

42. Mao S, Shi J, Sun G, Ma D, He C, Pu Z, et al. Au nanodots@ thiol-UiO66@ZnIn2S4 nanosheets with significantly enhanced visible-light photocatalytic $\mathrm{H} 2$ evolution: the effect of different Au positions on the transfer of electron-hole pairs. Appl Catal B. $2021 ; 282: 119550$.

43. Wang K, Xing Z, Meng D, Zhang S, Li Z, Pan K, et al. Hollow MoSe2@Bi2S3/CdS Core-Shell nanostructure as dual Z-Scheme heterojunctions with enhanced full spectrum photocatalyticphotothermal performance. Appl Catal B. 2021;281:119482. 
44. Onkani SP, Diagboya PN, Mtunzi FM, Klink MJ, Olu-Owolabi BI, Pakade V. Comparative study of the photocatalytic degradation of 2-chlorophenol under UV irradiation using pristine and Agdoped species of $\mathrm{TiO} 2, \mathrm{ZnO}$ and $\mathrm{ZnS}$ photocatalysts. J Environ Manage. 2020;260:110145.

45. Ismael M. Enhanced photocatalytic hydrogen production and degradation of organic pollutants from $\mathrm{Fe}$ (III) doped $\mathrm{TiO} 2$ nanoparticles. J Environ Chem Eng. 2020;8(2):103676.

46. Qi K, Xing X, Zada A, Li M, Wang Q, Liu S-y, et al. Transition metal doped $\mathrm{ZnO}$ nanoparticles with enhanced photocatalytic and antibacterial performances: experimental and DFT studies. Ceram Int. 2020;46(2):1494-502.

47. Kresse F. Efficient iterative schemes for ab initio total-energy calculations using a plane-wave basis set. Phys Rev B Condens Matter. 1996;54(16):11169-86.

48. Hohenberg P, Kohn W. Inhomogeiieous electron gas. Phys Rev. 1964;136(3B):864-71.

49. Kohn W, Sham LJ. Self-consistent equations including exchange and correlation effects. Phys Rev. 1965;140:A1133.

50. Perdew JP, Chevary JA, Vosko SH, Jackson KA, Pederson MR, Singh DJ, et al. Atoms, molecules, solids, and surfaces: applications of the generalized gradient approximation for exchange and correlation. Phys Rev B Condens Matter. 1992;46(11):6671-87.

51. Perdew J, Burke K, Ernzerhof M. Generalized gradient approximation made simple. Phys Rev Lett. 1996;77:3865-8.

52. Mohamed MA, Zain MFM, Minggu LJ, Kassim MB, Jaafar J, Amin NAS, et al. Bio-inspired hierarchical hetero-architectures of in-situ C-doped g-C3N4 grafted on $\mathrm{C}, \mathrm{N}$ co-doped $\mathrm{ZnO}$ micro-flowers with booming solar photocatalytic activity. J Ind Eng Chem. 2019;77:393-407.

53. Zhang JY, Xue XZ, Liu JK. Eminently enhanced anticorrosion performance and mechanisms of $\mathrm{X}-\mathrm{ZnO}(\mathrm{X}=\mathrm{C}, \mathrm{N}$, and $\mathrm{P})$ solid solutions. Inorg Chem. 2017;56(20):12260-71.

54. Liang P, Zhang C, Sun HQ, Liu SM, Tade M, Wang SB. Photocatalysis of $\mathrm{C}, \mathrm{N}$-doped $\mathrm{ZnO}$ derived from $\mathrm{ZIF}-8$ for dye degradation and water oxidation. RSC Advances. 2016;6(98):95903-9.

55. Li P, Zhang W, Zhang X, Wang Z, Wang X, Ran S, et al. Synthesis, characterization, and photocatalytic properties of flower-like mn-doped ceria. Mater Res. 2018;21(5):1-6.

56. Dai B, Xuan M, Lv Y, Jin C, Ran S. Molten salt synthesis of Bi2WO6 powders and its visible-light photocatalytic activity. Mater Res. 2019;22(5):1-7.

57. Raja A, Rajasekaran P, Vishnu B, Selvakumar K, Yeon Do J, Swaminathan $\mathrm{M}$, et al. Fabrication of effective visible-lightdriven ternary Z-scheme $\mathrm{ZnO}-\mathrm{Ag}$-BiVO4 heterostructured photocatalyst for hexavalent chromium reduction. Separ Purif Tech. 2020;252:117446.
58. Zhao W, Li Y, Zhao P, Zhang L, Dai B, Xu J, et al. Novel Z-scheme Ag-C3N4/SnS2 plasmonic heterojunction photocatalyst for degradation of tetracycline and $\mathrm{H} 2$ production. Chem Eng J. 2021;405:126555.

59. Mumanga TJ, Díaz-Torres LA, Gómez-Solís C. Nd3+ doped $\mathrm{BaA} 2 \mathrm{O} 4$ for enhanced photocatalytic degradation of methylene blue. Mater Lett. 2021;292:129664.

60. Chahar D, Taneja S, Bisht S, Kesarwani S, Thakur P, Thakur A, et al. Photocatalytic activity of cobalt substituted zinc ferrite for the degradation of methylene blue dye under visible light irradiation. J Alloys Compd. 2021;851:156878.

61. Zheng J, Sun L, Jiao C, Shao Q, Lin J, Pan D, et al. Hydrothermally synthesized $\mathrm{Ti} / \mathrm{Zr}$ bimetallic MOFs derived N self-doped $\mathrm{TiO} 2 / \mathrm{ZrO} 2$ composite catalysts with enhanced photocatalytic degradation of methylene blue. Colloids Surf A Physicochem Eng Asp. 2021;623:126629.

62. Mosavi SA, Ghadi A, Gharbani P, Mehrizad A. Photocatalytic removal of Methylene Blue using Ag@CdSe/Zeoilte nanocomposite under visible light irradiation by Response Surface Methodology. Mater Chem Phys. 2021;267:124696.

63. Wang S, Gao H, Fang L, Hu Q, Sun G, Chen X, et al. Synthesis of novel CQDs/CeO2/SrFe12O19 magnetic separation photocatalysts and synergic adsorption-photocatalytic degradation effect for methylene blue dye removal. Adv Chem Eng. 2021;6:100089.

64. Cheng T, Chen C, Wang L, Zhang X, Ye C, Deng Q, et al. Synthesis of fly ash magnetic glass microsphere@BiVO4 and its hybrid action of visible-light photocatalysis and adsorption process. Pol J Environ Stud. 2021;30(3):2027-40.

65. Collins SM, Pearce AK, Ferreira KM, Fenwick AJ, Regan PH, Keightley JD. Direct measurement of the half-life of (223)Ra. Appl Radiat Isot. 2015;99:46-53.

66. Tang H, Fu Y, Chang S, Xie S, Tang G. Construction of Ag3PO4/ $\mathrm{Ag} 2 \mathrm{MoO} 4 \mathrm{Z}$-scheme heterogeneous photocatalyst for the remediation of organic pollutants. Chin J Catal. 2017;38(2):33747.

67. Zhu D, Zhou Q. Nitrogen doped g-C3N4 with the extremely narrow band gap for excellent photocatalytic activities under visible light. Appl Catal B. 2021;281:119474.

68. Kim D, Yong K. Boron doping induced charge transfer switching of a $\mathrm{C} 3 \mathrm{~N} 4 / \mathrm{ZnO}$ photocatalyst from Z-scheme to type II to enhance photocatalytic hydrogen production. Appl Catal B. 2021;282:119538.

69. Wong KT, Kim SC, Yun K, Choong CE, Nah IW, Jeon $\mathrm{B}-\mathrm{H}$, et al. Understanding the potential band position and $\mathrm{e}-\mathrm{h}(+)$ separation lifetime for Z-scheme and type-II heterojunction mechanisms for effective micropollutant mineralization: comparative experimental and DFT studies. Appl Catal B. 2020;273:119034. 